\section{Prevalence of dental trauma in Brazilian children and adolescents: a systematic review and meta-analysis}

\section{Prevalência de lesões dentárias traumáticas em crianças e adolescentes brasileiros: uma revisão sistemática e metanálise}

\section{Prevalencia de lesiones dentales traumáticas en niños brasileños y adolescentes: una revisión sistemática y metaanálisis}

Walbert de Andrade Vieira 1

Vanessa Gallego Arias Pecorari 2 Rodolfo Figueiredo-de-Almeida 1

Nelson Carvas Junior 2

Júlio Vargas-Neto 1

Eduardo César Almada Santos 1

Brenda Paula Figueiredo de Almeida Gomes 1

José Flávio Affonso de Almeida 1

Adriana de-Jesus-Soares 1

\begin{abstract}
This study aimed to investigate the prevalence of dental trauma in Brazilian children and adolescents. A systematic review was conducted considering eight databases: MEDLINE (via PubMed), LILACS, BBO, Scopus, Embase, Web of Science, Open Access Theses and Dissertations, and OpenThesis. Only prevalence studies that used a probabilistic sampling method were included, without restriction on year or language of publication. The JBI critical appraisal tools for prevalence studies were used to assess the individual risk of bias. The individual studies were combined in the meta-analysis using the random-effects model. The heterogeneity between the studies was analyzed by Cochran's $Q$ and the I-square statistics. A meta-regression analysis was performed to evaluate the sources of heterogeneity. The GRADE approach assessed the certainty of evidence across included studies. The search resulted in 2,069 records, of which 36 were included in the study. The eligible studies were published from 2000 to 2021, with a total sample of 40,194 children and adolescents. Most studies (75\%) had a low risk of bias. In permanent teeth, the prevalence of dental trauma was $21 \%$ (95\% CI: 16.0; 26.0) and in deciduous teeth; 35\% (95\%CI: 26.0; 44.0). The prevalence of dental trauma among boys was higher than among girls for both dentitions. Based on a low certainty, the prevalence of traumatic dental injuries in Brazilian children and adolescents is higher than that found worldwide both in deciduous and permanent teeth. Also, the prevalence of dental trauma among boys is higher than among girls.
\end{abstract}

Tooth Injuries; Child; Adolescent; Systematic Review
Correspondence

W. A. Vieira

Departamento de Odontologia Restauradora, Faculdade de Odontologia de Piracicaba, Universidade Estadual de Campinas. Rua Prudente de Morais 261, Piracicaba, SP 13400-310, Brasil walbert.vieira18@gmail.com

1 Faculdade de Odontologia de Piracicaba, Universidade
Estadual de Campinas, Piracicaba, Brasil.

2 Universidade Paulista, Piracicaba, Brasil. 


\section{Introduction}

The International Association of Dental Traumatology (IADT) defines dental trauma as an external impact on dental tissue and may present itself clinically as an injury to hard (enamel fracture, enamel fracture and dentin with or without pulp exposure, root fracture, and alveolar fracture) or supportive (concussion, subluxation, intrusive, extrusive, or lateral dislocation, and avulsion) tissues of the teeth 1 . The dental trauma is a worldwide public health problem and can be considered the fifth most common group of injuries in the world 2. Children and adolescents are the most affected by dental trauma, with an estimated average prevalence of $18 \%$ among 12 -years-old 3,4. The consequences of dental trauma in this age group can be presented in different ways, such as a decreased quality of life 5 , tooth loss 6 , pulp necrosis followed by root resorption 7 , or interruption of root formation 8 ; and additionally, emotional stress for both child and parents 9 .

In Brazil, an epidemiological survey performed in 2010 with adolescents aged 12 year found that the prevalence of pediatric dental trauma was $21.5 \% 10$. However, the survey did not include children in primary dentition, who also have a high prevalence of pediatric dental trauma ${ }^{3}$. This study was carried out in 2010, and it has not been updated since then. Recently, Aldrigui et al. ${ }^{4}$ published a systematic review and meta-analysis assessing the prevalence of pediatric dental trauma in Latin America and the Caribbean. However, this systematic review has only included a few Brazilian studies, and yet no subgroup analysis for the population was performed. Furthermore, the bibliographic search was carried out in 2011; and since then, several Brazilian studies have been published.

Moreover, Brazil is a large country, in which its prevalence rates of pediatric dental trauma may vary according to each region 11,12,13,14,15. This inconsistency may be a result of socioeconomic, behavioral, and phenotypic differences, but also of the lack of information on the epidemiology of dental trauma in certain sparsely populated areas. Thus, knowing the epidemiological profile of pediatric dental trauma in Brazil and its regions might help to understand the proportion and impact of these injuries on the population as well as helping to identify areas that need to implement more urgent preventive measures.

Thus, although most epidemiological pediatric dental trauma studies in the literature have been conducted in Brazil 3, no meta-analysis has determined the prevalence of pediatric dental trauma in the population. Then, this systematic review aimed to answer the following guiding question: "What is the prevalence of dental trauma in children and adolescents in Brazil?".

\section{Methods}

\section{Protocol and registration}

A protocol was registered at the International Prospective Register of Systematic Reviews (PROSPERO) database, under the number CRD42018111212. This systematic review was reported according to the Preferred Reporting Items for Systematic Reviews and Meta-Analyses (PRISMA) 16 and was conducted according to the Joanna Briggs Institute (JBI) manual 17.

The original protocol (published in October 2018) was designed to investigate the prevalence and risk factors associated with dental trauma in Brazilian children and adolescents; however, due to a large number of variables, the authors chose to continue the review focusing only on epidemiological prevalence data - this decision was made before the study selection process. Then, a new update in October 2019 was carried out to include this change and adaptations of tools for assessing the risk of bias and meta-analysis models that would be used.

\section{Eligibility criteria}

The inclusion and exclusion criteria were based on the CoCoPop mnemonic (Condition, Context, and Population) 17. 


\section{Inclusion criteria}

\section{- Condition}

Diagnosis of pediatric dental trauma in the anterior teeth (maxillary or mandibular) by Andreasen's or Obrien's criteria. Both criteria considered only the clinical appearance of the dental trauma, as crown color change, fracture involving enamel, fracture involving enamel and dentin, fracture involving enamel, dentin, and pulp, absence and restoration due to trauma.

\section{- Context}

Studies performed in Brazil.

\section{- Population}

Brazilian children and adolescents aged 0-19 years, regardless of sex, ethnicity, or other sociodemographic factors. The 19-year-old threshold was established according to the classification of the World Health Organization (WHO) for "adolescent" 18.

\section{- Study design}

We considered only prevalence studies that used a probabilistic sampling method (simple or complex) or that included all target populations.

\section{Exclusion criteria}

The following studies were excluded: (1) studies performed in emergency and traumatology services, where pediatric dental trauma frequency could be artificially higher; (2) studies including participants presenting a cleft lip and/or palate as well as other craniofacial deformities or any syndrome; (3) studies with overlapping results; in this case, we considered the most recent study with the best methodological quality.

\section{Sources of information and search}

Embase, MEDLINE (via PubMed), Scopus, Web of Science, LILACS, and BBO databases were used as primary study sources. OpenGrey and Open Access Theses and Dissertations were used to partially capture the "gray literature". All steps were performed to minimize selection and publication biases.

The MeSH (Medical Subject Headings), DeCS (Health Sciences Descriptors), and Emtree (Embase Subject Headings) resources were used to select appropriate search descriptors. The Boolean operators "AND" and "OR" were used to enhance the research strategy through several combinations (Supplementary Material. Box S1. http://cadernos.ensp.fiocruz.br/static//arquivo/suppl-e00015920_9970. pdf). The bibliographic search was performed in January, 2019, and updated on February 2nd, 2021. The search strategy included the following descriptors: "tooth injuries", "tooth avulsion," "maxillofacial injuries", "Brazil". Also, the following DeCS descriptors: "traumatismos dentários" [Portuguese], “avulsão dentária” [Portuguese], "Brasil” [Portuguese]. The search strategy was adapted for each database, respecting their rules of syntax.

The results obtained were exported to the EndNote Web software (https://endnote.com/), in which duplicates were considered only once.

\section{Study selection}

Study selection was performed in three phases. In the first phase, as a calibration exercise, two reviewers discussed the eligibility criteria and applied them to a sample of $20 \%$ of the studies retrieved after the initial search to determine inter-rater agreement. After achieving a proper level of agreement 
(kappa $\geq 0.81$ ), two reviewers (W.A.V. and R.F.A.) performed a methodical analysis of all the titles of the studies, independently. The reviewers were not blind to the names of authors and journals. In the second phase, the reviewers (W.A.V. and R.F.A.) read the abstracts independently for the initial application of the exclusion criteria. Studies containing titles that met the objectives of the study but did not have abstracts available were fully read in phase three.

In the third phase, preliminary eligible studies had their full texts evaluated to verify whether they fulfilled the eligibility criteria. When both reviewers disagreed, a third reviewer (A.J.S.) was consulted to make a final decision. Excluded studies were registered in a separate database listing the reasons for exclusion.

\section{Data collection}

After the selection, the studies were analyzed and two reviewers (W.A.V. and R.F.A.) extracted information regarding the identification of the study (author, year, city, state, and region where the research was conducted, funding sources), sample characteristics (number of patients, distribution by sex, age range, and sample collection location), characteristics of data collection (evaluation period, trauma diagnosis criteria), and main results (overall prevalence and prevalence by gender). If prevalence were not directly provided, they were calculated. In case of missing data, we contacted the corresponding author by e-mail.

A calibration exercise was performed with both reviewers (W.A.V. and R.F.A.) in order to ensure consistency among reviewers, in which information was extracted jointly from an eligible study. Any disagreement between the reviewers was solved through discussions, and when both reviewers disagreed, a third one (A.J.S.) was consulted to make a final decision.

\section{Risk of bias in individual studies}

Two authors (W.A.V. and R.F.A.) assessed the risk of bias and individual quality of the studies selected, independently, using the JBI critical appraisal tools for use in JBI systematic reviews 17 for prevalence studies. As a calibration purpose, the authors analyzed an eligible study jointly, with the presence of a third reviewer (A.J.S.) in charge of solving divergences in case of doubts.

This tool is composed of nine questions, as follows: (1) "Was the sample frame appropriate to address the target population?", (2) "Were the study participants sampled appropriately?", (3) "Was the sample size adequate?", (4) "Were the study subjects and the setting described in detail?", (5) "Was the data analysis conducted with sufficient coverage of the identified sample?", (6) "Were valid methods used for the identification of the condition?", (7) "Was the condition measured in a standard, reliable way for all participants?", (8) "Was there appropriate statistical analysis?" and (9) "Was the response rate adequate, and if not, was the low response rate managed appropriately?".

Each question could be answered as: "yes" - if the study did not present bias regarding the domain evaluated by the question; or "no" - if the study presented bias regarding the domain evaluated by the question; or "unclear" - if the study did not provide sufficient information to evaluate the bias in the question; or (4) "not applicable" - if the question was not suitable for the study.

The individual bias of the study was categorized according to the sum of positive answers to the questions corresponding to the assessment tool. The risk of bias was considered high when the study obtained less than 4 of "yes" answers, moderate when the study obtained 5 to 7 of "yes" answers, and low when the study reached 8 or 9 of "yes" scores 19,20,21.

\section{Summary measures and syntheses of results}

To give equal weights to the different populations investigated, only one study per age in each municipality was included in the meta-analysis. For this purpose, we selected the most recent study with the best methodology for each municipality. Studies conducted in the same municipality but evaluating different age groups of children or adolescents were included in the meta-analysis.

The analyses were conducted considering the type of teeth (permanent or deciduous) evaluated in the studies. The individual studies were combined in the meta-analysis using the random-effects 
model proposed by Dersimonian-Laird and Freeman-Tukey double arcsine transformation. The heterogeneity between the studies was analyzed by Cochran's Q statistics and its magnitude was estimated by the I-square (I2) statistics. For each analysis, the data were grouped into subgroups considering two variables: (1) the regions of Brazil and (2) the gender of the sample.

The causes of heterogeneity were assessed by meta-regression, evaluating the effect of the following variables: year of publication, average age of the sample, population size, Human Development Index (HDI), and trauma diagnostic criteria. The data regarding the HDI and population size of each city were collected from the online system of the Brazilian Institute of Geography and Statistics (IBGE. https://cidades.ibge.gov.br/).

The existence of the small-study effect was also assessed by visual inspection of the funnel graph and the Egger test. All analyses were performed in the R program version 4.0 (https://www.rproject.org/).

\section{Certainty of evidence collection}

Certainty of the identified evidence was assessed with the Grading of Recommendation, Assessment, Development, and Evaluation (GRADE) tool 22 with adaptations proposed by Iorio et al. 23. For meta-analysis of prevalence, the best evidence is obtained through cross-sectional studies or baseline examination from cohort studies. Thus, evidence derived from these types of studies initiates the assessment as having "high certainty of evidence," and can be downgraded by the risk of bias, inconsistency, indirectness, imprecision, and publication bias. Finally, the level of certainty among the identified evidence can be characterized as high, moderate, low, or very low 22.

\section{Results}

\section{Study selection}

During the first phase of the study selection, 2,069 results were found distributed in eight electronic databases, including the gray literature. After removing the duplicates, 1,182 results remained for the analysis of titles and abstracts. After applying the eligibility criteria to the titles and abstracts, 126 results were eligible for the full-text analysis. After reading the full-text, 90 studies were excluded (Supplementary Material. Box S2. http://cadernos.ensp.fiocruz.br/static//arquivo/supple00015920_9970.pdf). Thus, 36 studies 14,24,25,26,27,28,29,30,31,32,33,34,35,36,37,38,39,40,41,42,43,44,45,46,47,48,49, $50,51,52,53,54,55,56,57,58$ were selected for the qualitative analysis (Figure 1).

\section{Characteristics of eligible studies}

The studies were published between 2000 and 2021, and most studies were conducted in the Southern (16 studies) 42,43,44,45,46,47,48,49,50,51,52,53,54,55,56,57 and Southeastern (13 studies) 14,30,31,32,33,34,35,36,37,38,39 $, 40,41$ regions, and one study was conducted in all regions 58 . The total sample consisted of 40,194 children and adolescents aged from 1 to 19 years. Fifteen studies 25,26,27,31,33,34,35,38,40,41,48,51,52,55,56 evaluated the prevalence of pediatric dental trauma in deciduous teeth and 21 studies 14,24,28,29,30,32,36,37, $39,42,43,44,45,46,47,49,50,53,54,57,58$ evaluated the prevalence of pediatric dental trauma in permanent teeth.

Four studies included all target populations in the study, one study used a stratified multi-phase cluster sampling method, and the other studies used a single, two, or three-phase random sampling method (Table 1). The assessment of the prevalence of dental trauma was conducted through clinical examination mostly in public and private schools along with health centers on national child vaccination days. The most used diagnostic criterion among studies was Andreasen's, used by 55\% of the studies, followed by the criterion established by O'Brien, used by $45 \%$ of the studies. The number of examiners in each study varied from 1 to 18 . Four of the studies 33,41,48,49 did not make it clear how many examiners have performed the clinical evaluations. 
Figure 1

Flowchart of the process of literature search and selection, adapted from the PRISMA statement.

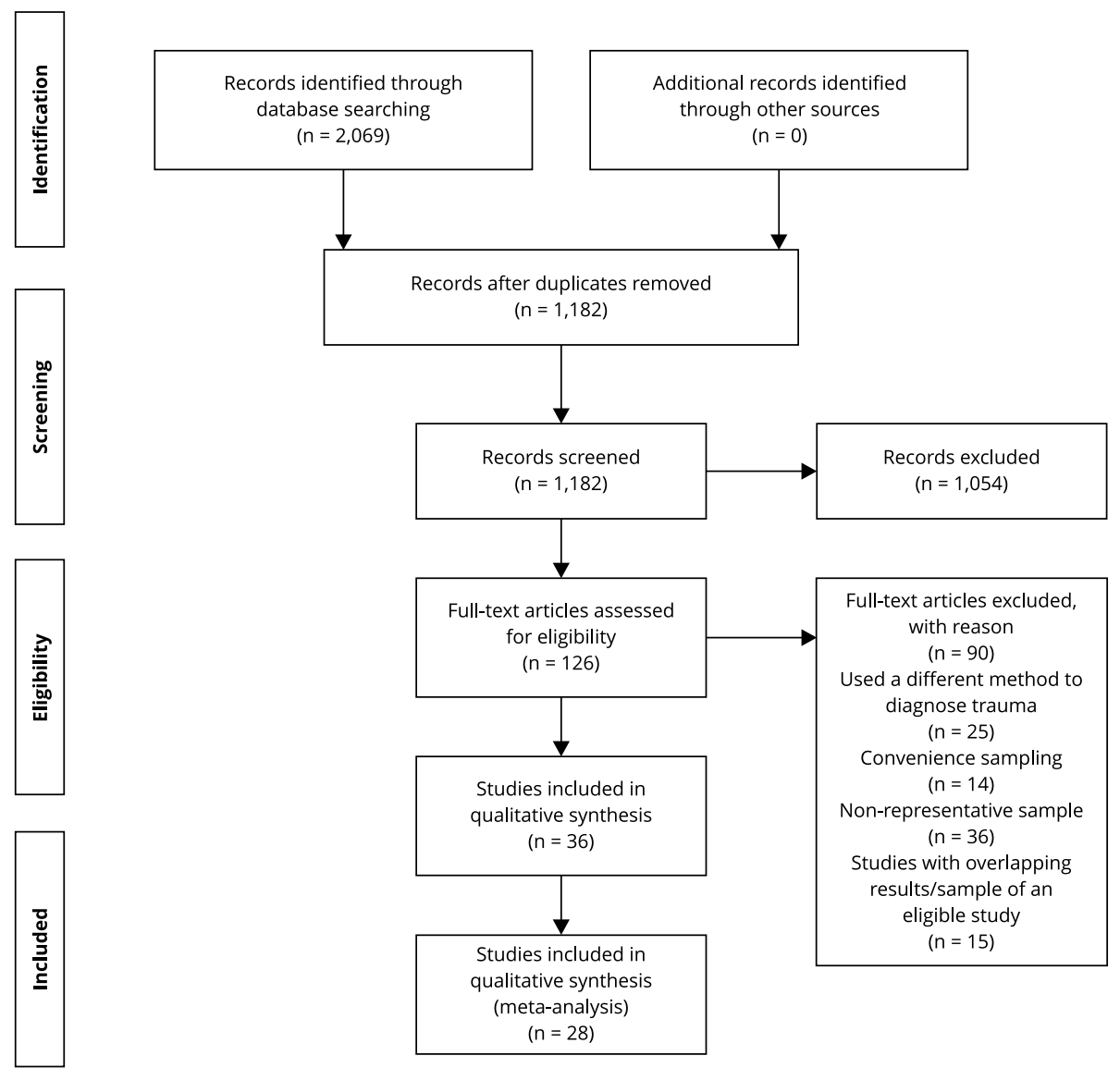

\section{Risk of bias in individual studies}

Among the 36 studies included, none were scored 4 or less, nine studies (25\%) scored 4 to 7 , and the others (75\%) scored 8 or 9 . The main shortcomings were related to the lack of details of the sampling method (19.4\% of the studies) and the use of an unappropriated statistical analysis (63.9\% of the studies) (Supplementary Material. Table S1. http://cadernos.ensp.fiocruz.br/static//arquivo/supple00015920_9970.pdf).

For question 2, those studies that encompassed the entire population were classified as "yes". The studies that used a random sampling method and showed all detailed information - the precision of the final prevalence estimation which incorporates the clustering effect and details of randomization - were classified as "yes", while those that presented incomplete information were classified as "unclear".

Regarding the method of assessing the prevalence of dental trauma (question 7), studies that did not describe the number of operators and whether calibration was performed (kappa test and calibration method) were classified as "no". In contrast, the studies that described the number of operators and stated that calibration was performed, but without details about the kappa test and calibration method, were classified as "unclear". 
Table 1

Main characteristics of the eligible studies.

\begin{tabular}{|c|c|c|c|c|c|c|c|c|c|c|}
\hline $\begin{array}{l}\text { Region/ } \\
\text { Study (year } \\
\text { of publi- } \\
\text { cation) }\end{array}$ & City (State) & $\begin{array}{l}\text { Sampling } \\
\text { method }\end{array}$ & $\begin{array}{l}\text { Represen- } \\
\text { tativity }\end{array}$ & $\begin{array}{c}\text { Sample } \\
\text { analyzed (n) }\end{array}$ & Setting & $\begin{array}{l}\text { Number } \\
\text { of raters }\end{array}$ & $\begin{array}{c}\text { Dental } \\
\text { trauma } \\
\text { diagnostic } \\
\text { classifi- } \\
\text { cation } \\
\text { criteria }\end{array}$ & $\begin{array}{c}\text { Year of } \\
\text { data } \\
\text { colle- } \\
\text { ction }\end{array}$ & $\begin{array}{c}\text { Prevalence } \\
\text { of dental } \\
\text { trauma }\end{array}$ & $\begin{array}{l}\text { Funding } \\
\text { source }\end{array}$ \\
\hline \multicolumn{11}{|l|}{ Northeast } \\
\hline $\begin{array}{l}\text { Soriano } \\
\text { et al. } 24 \\
(2009)\end{array}$ & Recife (PE) & $\begin{array}{l}\text { Single- } \\
\text { phase } \\
\text { random } \\
\text { sampling * }\end{array}$ & $\begin{array}{l}12 \text { years-old } \\
\text { school children } \\
\text { living in the } \\
\text { whole city }\end{array}$ & $\begin{array}{c}1,046 \\
\left(520 \% 526{ }^{\Uparrow}\right)\end{array}$ & $\begin{array}{l}\text { Public and } \\
\text { private } \\
\text { schools }\end{array}$ & 1 & Andreasen & 2002 & $\begin{array}{c}10.5 \% \\
(110 / 1,046)\end{array}$ & + \\
\hline $\begin{array}{l}\text { Siqueira } \\
\text { et al. } 25 \\
\text { (2013) }\end{array}$ & $\begin{array}{l}\text { Campina } \\
\text { Grande (PB) }\end{array}$ & $\begin{array}{l}\text { Two-phase } \\
\text { random } \\
\text { sampling ** }\end{array}$ & $\begin{array}{l}\text { 3-5 years-old } \\
\text { preschool } \\
\text { children living in } \\
\text { the whole city }\end{array}$ & $\begin{array}{c}814 \\
\left(392+422{ }^{\Uparrow}\right)\end{array}$ & $\begin{array}{l}\text { Public and } \\
\text { private } \\
\text { schools }\end{array}$ & 3 & Andreasen & 2012 & $\begin{array}{c}34.5 \% \\
(281 / 814)\end{array}$ & $\begin{array}{c}\text { CAPES, } \\
\text { FAPEMIG } \\
\text { and CNPq }\end{array}$ \\
\hline $\begin{array}{l}\text { Gomes } \\
\text { et al. } 26 \\
\text { (2014) }\end{array}$ & $\begin{array}{l}\text { Campina } \\
\text { Grande (PB) }\end{array}$ & $\begin{array}{l}\text { Two-phase } \\
\text { random } \\
\text { sampling ** }\end{array}$ & $\begin{array}{l}\text { 3-5 years-old } \\
\text { preschool } \\
\text { children living in } \\
\text { the whole city }\end{array}$ & $\begin{array}{c}843 \\
(405+434 \lesssim)\end{array}$ & $\begin{array}{l}\text { Public and } \\
\text { private } \\
\text { schools }\end{array}$ & 3 & Andreasen & + & $\begin{array}{c}34.1 \% \\
(286 / 843)\end{array}$ & $\begin{array}{c}\text { CAPES, } \\
\text { FAPEMIG } \\
\text { and CNPq }\end{array}$ \\
\hline $\begin{array}{l}\text { Neves } \\
\text { et al. } 27 \\
(2017)\end{array}$ & $\begin{array}{l}\text { Campina } \\
\text { Grande (PB) }\end{array}$ & $\begin{array}{l}\text { Two-phase } \\
\text { random } \\
\text { sampling ** }\end{array}$ & $\begin{array}{l}5 \text { years-old } \\
\text { preschool } \\
\text { children living in } \\
\text { the whole city }\end{array}$ & $\begin{array}{c}769 \\
\left(366+403{ }^{\Uparrow}\right)\end{array}$ & $\begin{array}{l}\text { Public and } \\
\text { private } \\
\text { schools }\end{array}$ & 2 & Andreasen & 2015 & $\begin{array}{c}54.3 \% \\
(407 / 769)\end{array}$ & $\begin{array}{c}\text { CAPES, } \\
\text { FAPEMIG } \\
\text { and CNPq }\end{array}$ \\
\hline $\begin{array}{l}\text { Carvalho } \\
\text { et al. } 28 \\
(2020)\end{array}$ & Recife (PE) & $\begin{array}{l}\text { Two-phase } \\
\text { random } \\
\text { sampling ** }\end{array}$ & $\begin{array}{l}\text { 15-19 years- } \\
\text { old school } \\
\text { adolescents } \\
\text { living in the } \\
\text { whole city }\end{array}$ & $\begin{array}{c}1,485 \\
(823+662 \precsim)\end{array}$ & $\begin{array}{l}\text { Public and } \\
\text { private } \\
\text { schools }\end{array}$ & 1 & Andreasen & 2009 & $\begin{array}{c}17.8 \% \\
(264 / 1,485)\end{array}$ & CAPES \\
\hline \multicolumn{11}{|l|}{ Central-West } \\
\hline $\begin{array}{l}\text { Freire et al. } 29 \\
(2014)\end{array}$ & $\begin{array}{c}\text { Goiania } \\
\text { (GO) }\end{array}$ & $\begin{array}{l}\text { Two-phase } \\
\text { random } \\
\text { sampling ** }\end{array}$ & $\begin{array}{l}12 \text { years-old } \\
\text { school children } \\
\text { living in the } \\
\text { whole city }\end{array}$ & $\begin{array}{c}2,075 \\
\left(1,053 \propto 1,022{ }^{\jmath}\right)\end{array}$ & $\begin{array}{l}\text { Public and } \\
\text { private } \\
\text { schools }\end{array}$ & 6 & O'Brien & 2010 & $\begin{array}{c}17.2 \% \\
(358 / 2,075)\end{array}$ & FAPEG \\
\hline \multicolumn{11}{|l|}{ Southeastern } \\
\hline $\begin{array}{l}\text { Cortes } \\
\text { et al. } 30 \\
(2001)\end{array}$ & $\begin{array}{c}\text { Belo } \\
\text { Horizonte } \\
\text { (MG) }\end{array}$ & $\begin{array}{c}\text { Two-phase } \\
\text { random } \\
\text { sampling } \\
\star \star \star \star\end{array}$ & $\begin{array}{l}\text { 9-14 years-old } \\
\text { school children } \\
\text { living in the } \\
\text { whole city }\end{array}$ & $\begin{array}{c}3,702 \\
\left(1,973 \ngtr 1,729{ }^{\prime}\right)\end{array}$ & $\begin{array}{l}\text { Public and } \\
\text { private } \\
\text { schools }\end{array}$ & 1 & O'Brien & + & $\begin{array}{c}12.1 \% \\
(448 / 3,702)\end{array}$ & CNPq \\
\hline $\begin{array}{l}\text { Dutra et al. } 31 \\
(2010)\end{array}$ & $\begin{array}{l}\text { Matozinhos } \\
\text { (MG) }\end{array}$ & $\begin{array}{l}\text { Single- } \\
\text { phase } \\
\text { random } \\
\text { sampling \# }\end{array}$ & $\begin{array}{l}\text { 1-4 years-old } \\
\text { preschool } \\
\text { children living in } \\
\text { the whole city }\end{array}$ & $\begin{array}{c}407 \\
(202 \bigcirc 205 \precsim)\end{array}$ & $\begin{array}{l}\text { National } \\
\text { Child } \\
\text { Vaccination } \\
\text { Day }\end{array}$ & 5 & Andreasen & 2008 & $\begin{array}{c}45.9 \% \\
(187 / 407)\end{array}$ & + \\
\hline $\begin{array}{l}\text { Jorge et al. } 32 \\
(2012)\end{array}$ & $\begin{array}{c}\text { Belo } \\
\text { Horizonte } \\
\text { (MG) }\end{array}$ & $\begin{array}{l}\text { Two-phase } \\
\text { random } \\
\text { sampling \#\# }\end{array}$ & $\begin{array}{l}15-19 \text { years } \\
\text { old school } \\
\text { adolescents } \\
\text { living in the } \\
\text { whole city }\end{array}$ & $\begin{array}{c}891 \\
\left(539+352{ }^{\Uparrow}\right)\end{array}$ & $\begin{array}{l}\text { Public and } \\
\text { private } \\
\text { schools }\end{array}$ & 2 & Andreasen & 2009 & $\begin{array}{c}24.7 \% \\
(220 / 891)\end{array}$ & + \\
\hline
\end{tabular}

(continues) 
Table 1 (continued)

\begin{tabular}{|c|c|c|c|c|c|c|c|c|c|c|}
\hline $\begin{array}{l}\text { Region/ } \\
\text { Study (year } \\
\text { of publi- } \\
\text { cation) }\end{array}$ & City (State) & $\begin{array}{l}\text { Sampling } \\
\text { method }\end{array}$ & $\begin{array}{l}\text { Represen- } \\
\text { tativity }\end{array}$ & $\begin{array}{c}\text { Sample } \\
\text { analyzed (n) }\end{array}$ & Setting & $\begin{array}{l}\text { Number } \\
\text { of raters }\end{array}$ & $\begin{array}{c}\text { Dental } \\
\text { trauma } \\
\text { diagnostic } \\
\text { classifi- } \\
\text { cation } \\
\text { criteria }\end{array}$ & $\begin{array}{l}\text { Year of } \\
\text { data } \\
\text { colle- } \\
\text { ction }\end{array}$ & $\begin{array}{c}\text { Prevalence } \\
\text { of dental } \\
\text { trauma }\end{array}$ & $\begin{array}{l}\text { Funding } \\
\text { source }\end{array}$ \\
\hline $\begin{array}{l}\text { Bonini } \\
\text { et al. } 33 \\
(2012)\end{array}$ & Amparo (SP) & $\begin{array}{l}\text { Single- } \\
\text { phase } \\
\text { random } \\
\text { sam- } \\
\text { pling \#\#\# }\end{array}$ & $\begin{array}{l}\text { 3-4 years old } \\
\text { preschool } \\
\text { children living in } \\
\text { the city }\end{array}$ & $\begin{array}{c}376 \\
(191+185 \precsim)\end{array}$ & $\begin{array}{l}\text { National } \\
\text { Child } \\
\text { Vaccination } \\
\text { Day }\end{array}$ & 11 & Andreasen & + & $\begin{array}{c}27.7 \% \\
(104 / 376)\end{array}$ & + \\
\hline $\begin{array}{l}\text { Ramos-Jorge } \\
\text { et al. } 34 \\
(2013)\end{array}$ & $\begin{array}{c}\text { Belo } \\
\text { Horizonte } \\
\text { (MG) }\end{array}$ & $\begin{array}{l}\text { Single- } \\
\text { phase } \\
\text { random } \\
\text { sampling \# }\end{array}$ & $\begin{array}{l}\text { 1-3 years old } \\
\text { preschool } \\
\text { children living in } \\
\text { the whole city }\end{array}$ & $\begin{array}{c}519 \\
(249+270 \precsim)\end{array}$ & $\begin{array}{c}\text { National } \\
\text { Child } \\
\text { Vaccination } \\
\text { Day }\end{array}$ & 18 & Andreasen & + & $\begin{array}{c}41.2 \% \\
(214 / 519)\end{array}$ & + \\
\hline $\begin{array}{l}\text { Castro } \\
\text { et al. } 35 \\
(2013)\end{array}$ & $\begin{array}{c}\text { Casa Branca } \\
\text { (SP) }\end{array}$ & $\begin{array}{l}\text { All target } \\
\text { population } \\
\text { was } \\
\text { included }\end{array}$ & $\begin{array}{l}\text { 0-6 years old } \\
\text { preschool } \\
\text { children living in } \\
\text { the whole city }\end{array}$ & $\begin{array}{c}61 \\
(29+32 \precsim)\end{array}$ & + & 1 & Andreasen & $\begin{array}{l}2011- \\
2012\end{array}$ & $\begin{array}{c}1.6 \\
(1 / 61)\end{array}$ & + \\
\hline $\begin{array}{l}\text { Bendo } \\
\text { et al. } 36 \\
(2014)\end{array}$ & $\begin{array}{c}\text { Belo } \\
\text { Horizonte } \\
\text { (MG) }\end{array}$ & $\begin{array}{l}\text { Three-phase } \\
\text { random } \\
\text { sampling § }\end{array}$ & $\begin{array}{l}11-14 \text { years old } \\
\text { school children } \\
\text { living in the } \\
\text { whole city }\end{array}$ & $\begin{array}{c}1,122 \\
(667 \bigcirc 455 \precsim)\end{array}$ & $\begin{array}{l}\text { Public and } \\
\text { private } \\
\text { schools }\end{array}$ & 3 & Andreasen & 2009 & $\begin{array}{c}14.8 \% \\
(166 / 1,122)\end{array}$ & $\begin{array}{c}\text { CAPES and } \\
\text { FAPEMIG }\end{array}$ \\
\hline $\begin{array}{l}\text { Oliveira Filho } \\
\text { et al. } 37 \\
(2014)\end{array}$ & $\begin{array}{c}\text { Diamantina } \\
\text { (MG) }\end{array}$ & $\begin{array}{l}\text { Single- } \\
\text { phase } \\
\text { random } \\
\text { sampling } \S \S\end{array}$ & $\begin{array}{l}14-19 \text { years } \\
\text { old school } \\
\text { adolescents } \\
\text { living in the } \\
\text { whole city }\end{array}$ & $\begin{array}{c}687 \\
(389+298 \precsim)\end{array}$ & $\begin{array}{l}\text { Public and } \\
\text { private } \\
\text { schools }\end{array}$ & 1 & Andreasen & 2010 & $\begin{array}{c}26.6 \% \\
(183 / 687)\end{array}$ & + \\
\hline $\begin{array}{l}\text { Viegas } \\
\text { et al. } 38 \\
(2014)\end{array}$ & $\begin{array}{c}\text { Belo } \\
\text { Horizonte } \\
\text { (MG) }\end{array}$ & $\begin{array}{l}\text { Two-phase } \\
\text { random } \\
\text { sampling ** }\end{array}$ & $\begin{array}{l}5 \text { years old } \\
\text { preschool } \\
\text { children living in } \\
\text { the whole city }\end{array}$ & $\begin{array}{c}1,632 \\
(795+837 ð)\end{array}$ & $\begin{array}{l}\text { Public and } \\
\text { private } \\
\text { schools }\end{array}$ & 1 & Andreasen & 2009 & $\begin{array}{c}49.4 \% \\
(806 / 1,632)\end{array}$ & $\begin{array}{l}\text { CAPES, } \\
\text { CNPq and } \\
\text { FAPEMIG }\end{array}$ \\
\hline $\begin{array}{l}\text { Ramos-Jorge } \\
\text { et al. } 39 \\
(2014)\end{array}$ & $\begin{array}{c}\text { Diamantina } \\
\text { (MG) }\end{array}$ & $\begin{array}{l}\text { Two-phase } \\
\text { random } \\
\text { sampling ** }\end{array}$ & $\begin{array}{l}\text { 11-14 years old } \\
\text { school children } \\
\text { living in the } \\
\text { whole city }\end{array}$ & $\begin{array}{c}668 \\
(394+274 \precsim)\end{array}$ & $\begin{array}{l}\text { Public and } \\
\text { private } \\
\text { schools }\end{array}$ & 2 & O'Brien & 2012 & $\begin{array}{c}34.3 \% \\
(229 / 668)\end{array}$ & $\begin{array}{c}\text { CAPES and } \\
\text { FAPEMIG }\end{array}$ \\
\hline $\begin{array}{l}\text { Corrêa-Faria } \\
\text { et al. } 40 \\
\text { (2015) }\end{array}$ & $\begin{array}{c}\text { Diamantina } \\
\text { (MG) }\end{array}$ & $\begin{array}{c}\text { Single- } \\
\text { phase } \\
\text { systematic } \\
\text { sampling } \\
\text { §§§ }\end{array}$ & $\begin{array}{l}1-5 \text { years old } \\
\text { preschool } \\
\text { children living in } \\
\text { the whole city }\end{array}$ & $\begin{array}{c}301 \\
\left(145+156{ }^{\lambda}\right)\end{array}$ & $\begin{array}{l}\text { National } \\
\text { Child } \\
\text { Vaccination } \\
\text { Day }\end{array}$ & 10 & Andreasen & + & $\begin{array}{c}33.9 \% \\
(102 / 301)\end{array}$ & $\begin{array}{l}\text { CNPq and } \\
\text { FAPEMIG }\end{array}$ \\
\hline $\begin{array}{l}\text { Tello et al. } 41 \\
\text { (2016) }\end{array}$ & $\begin{array}{l}\text { Diadema } \\
\quad(\mathrm{SP})\end{array}$ & $\begin{array}{l}\text { Single- } \\
\text { phase } \\
\text { random } \\
\text { sampling † }\end{array}$ & $\begin{array}{l}\text { 1-4 years-old } \\
\text { preschool } \\
\text { children living in } \\
\text { the whole city }\end{array}$ & $\begin{array}{c}\text { 2002: } 779 \\
\text { 2004: } 925 \\
\text { 2006: } 1,014 \\
\text { 2008: } 1,198 \\
\text { 2010: } 1,258 \\
\text { 2012: } 1,215\end{array}$ & $\begin{array}{l}\text { National } \\
\text { Child } \\
\text { Vaccination } \\
\text { Day }\end{array}$ & + & Andreasen & $\begin{array}{l}2002 \\
2004 \\
2006 \\
2008 \\
2010 \\
2012\end{array}$ & 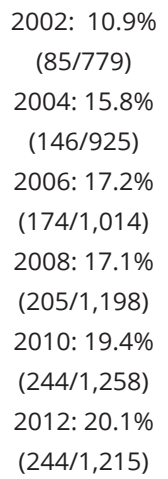 & $\begin{array}{l}\text { FAPESP, } \\
\text { CNPq and } \\
\text { CAPES }\end{array}$ \\
\hline
\end{tabular}

(continues) 
Table 1 (continued)

\begin{tabular}{|c|c|c|c|c|c|c|c|c|c|c|}
\hline $\begin{array}{l}\text { Region/ } \\
\text { Study (year } \\
\text { of publi- } \\
\text { cation) }\end{array}$ & City (State) & $\begin{array}{l}\text { Sampling } \\
\text { method }\end{array}$ & $\begin{array}{l}\text { Represen- } \\
\text { tativity }\end{array}$ & $\begin{array}{c}\text { Sample } \\
\text { analyzed (n) }\end{array}$ & Setting & $\begin{array}{l}\text { Number } \\
\text { of raters }\end{array}$ & $\begin{array}{c}\text { Dental } \\
\text { trauma } \\
\text { diagnostic } \\
\text { classifi- } \\
\text { cation } \\
\text { criteria }\end{array}$ & $\begin{array}{l}\text { Year of } \\
\text { data } \\
\text { colle- } \\
\text { ction }\end{array}$ & $\begin{array}{c}\text { Prevalence } \\
\text { of dental } \\
\text { trauma }\end{array}$ & $\begin{array}{c}\text { Funding } \\
\text { source }\end{array}$ \\
\hline $\begin{array}{l}\text { Freire-Maia et } \\
\text { al. } 14 \\
(2018)\end{array}$ & $\begin{array}{l}\text { Belo } \\
\text { Horizonte } \\
\text { (MG) }\end{array}$ & $\begin{array}{l}\text { Three-phase } \\
\text { random } \\
\text { sampling }{ }^{\dagger \dagger}\end{array}$ & $\begin{array}{l}\text { 8-10 years-old } \\
\text { school children } \\
\text { living in the } \\
\text { whole city }\end{array}$ & $\begin{array}{c}1.201 \\
(5369665 \gtrsim)\end{array}$ & $\begin{array}{l}\text { Public and } \\
\text { private } \\
\text { schools }\end{array}$ & 2 & Andreasen & 2010 & $\begin{array}{c}14.1 \% \\
(169 / 1,201)\end{array}$ & + \\
\hline \multicolumn{11}{|l|}{ South } \\
\hline $\begin{array}{l}\text { Marcenes } \\
\text { et al. } 42 \\
(2000)\end{array}$ & $\begin{array}{l}\text { Jaraguá do } \\
\text { Sul (SC) }\end{array}$ & $\begin{array}{l}\text { Two-phase } \\
\text { random } \\
\text { sampling t+† }\end{array}$ & $\begin{array}{l}12 \text { years-old } \\
\text { school children } \\
\text { living in the } \\
\text { whole city }\end{array}$ & $\begin{array}{c}476 \\
(225 \text { ㅇ } 251 \precsim)\end{array}$ & $\begin{array}{l}\text { Public and } \\
\text { private } \\
\text { schools }\end{array}$ & 1 & O'Brien & 1998 & $\begin{array}{c}15.3 \% \\
(73 / 476)\end{array}$ & + \\
\hline $\begin{array}{l}\text { Marcenes } \\
\text { et al. } 43 \\
(2001)\end{array}$ & $\begin{array}{l}\text { Blumenau } \\
\text { (SC) }\end{array}$ & $\begin{array}{l}\text { Two-phase } \\
\text { random } \\
\text { sampling t†t }\end{array}$ & $\begin{array}{l}12 \text { years-old } \\
\text { school children } \\
\text { living in the } \\
\text { whole city }\end{array}$ & $\begin{array}{c}652 \\
\left(329+323{ }^{\Uparrow}\right)\end{array}$ & $\begin{array}{l}\text { Public and } \\
\text { private } \\
\text { schools }\end{array}$ & 1 & O'Brien & 1998 & $\begin{array}{c}58.6 \% \\
(382 / 652)\end{array}$ & + \\
\hline $\begin{array}{l}\text { Nicolau } \\
\text { et al. } 44 \\
\text { (2003) }\end{array}$ & $\begin{array}{l}\text { Cianorte } \\
\text { (PR) }\end{array}$ & $\begin{array}{l}\text { All target } \\
\text { population } \\
\text { was } \\
\text { included }\end{array}$ & $\begin{array}{l}\text { All } 13 \text { years-old } \\
\text { children living in } \\
\text { the whole city }\end{array}$ & $\begin{array}{c}652 \\
(328+324 \lesssim)\end{array}$ & $\begin{array}{l}\text { Public and } \\
\text { private } \\
\text { schools }\end{array}$ & 1 & O'Brien & 1999 & $\begin{array}{c}20.4 \% \\
(133 / 652)\end{array}$ & CAPES \\
\hline $\begin{array}{l}\text { Traebert } \\
\text { et al. } 45 \\
(2004)\end{array}$ & Biguaçu (SC) & $\begin{array}{l}\text { All target } \\
\text { population } \\
\text { was } \\
\text { included }\end{array}$ & $\begin{array}{l}11-13 \text { years-old } \\
\text { children living in } \\
\text { the whole city }\end{array}$ & $\begin{array}{c}2,260 \\
\left(1,087 \uparrow 1,173{ }^{\lambda}\right)\end{array}$ & $\begin{array}{l}\text { Public and } \\
\text { private } \\
\text { schools }\end{array}$ & 1 & O'Brien & 2001 & $\begin{array}{c}10.7 \% \\
(242 / 2,260)\end{array}$ & + \\
\hline $\begin{array}{l}\text { Trabert } \\
\text { et al. } 46 \\
(2006)\end{array}$ & $\begin{array}{c}\text { Herval } \\
\text { D'Oeste (SC) }\end{array}$ & $\begin{array}{l}\text { All target } \\
\text { population } \\
\text { was } \\
\text { included }\end{array}$ & $\begin{array}{l}12 \text { years-old } \\
\text { children living in } \\
\text { the whole city }\end{array}$ & $\begin{array}{c}260 \\
\left(135+125{ }^{\lambda}\right)\end{array}$ & $\begin{array}{l}\text { Public and } \\
\text { private } \\
\text { schools }\end{array}$ & 1 & O'Brien & 2000 & $\begin{array}{c}17.3 \% \\
(45 / 260)\end{array}$ & + \\
\hline $\begin{array}{l}\text { Traebert } \\
\text { et al. } 47 \\
(2008)\end{array}$ & $\begin{array}{l}\text { Tubaração } \\
\text { (SC) }\end{array}$ & $\begin{array}{l}\text { Two-phase } \\
\text { random } \\
\text { sampling ** }\end{array}$ & $\begin{array}{l}\text { 7-8 years-old } \\
\text { school children } \\
\text { living in the } \\
\text { whole city }\end{array}$ & $\begin{array}{c}385 \\
(1949191 \precsim)\end{array}$ & $\begin{array}{l}\text { Public and } \\
\text { private } \\
\text { schools }\end{array}$ & 3 & O'Brien & 2003 & $\begin{array}{c}9.6 \% \\
(37 / 385)\end{array}$ & + \\
\hline $\begin{array}{l}\text { Kramer } \\
\text { et al. } 48 \\
(2009)\end{array}$ & Canela (RS) & $\begin{array}{l}\text { Single- } \\
\text { phase } \\
\text { random } \\
\text { sampling ₹ }\end{array}$ & $\begin{array}{l}\text { 0-5 years-old } \\
\text { preschool } \\
\text { children living in } \\
\text { the whole city }\end{array}$ & $\begin{array}{c}1,095 \\
(544+515 \gtrsim)\end{array}$ & $\begin{array}{l}\text { National } \\
\text { Child } \\
\text { Vaccination } \\
\text { Day }\end{array}$ & + & Andreasen & 2004 & $\begin{array}{c}23.6 \% \\
(258 / 1,095)\end{array}$ & + \\
\hline $\begin{array}{l}\text { Carvalho } \\
\text { et al. } 49 \\
(2010)\end{array}$ & Curitiba (PR) & $\begin{array}{l}\text { Two-phase } \\
\text { random } \\
\text { sampling } \neq \neq\end{array}$ & $\begin{array}{l}12-15 \text { years-old } \\
\text { children living in } \\
\text { the whole city }\end{array}$ & $\begin{array}{c}1,581 \\
(832+749 \precsim)\end{array}$ & $\begin{array}{l}\text { Public and } \\
\text { private } \\
\text { schools }\end{array}$ & + & O'Brien & $\begin{array}{c}2005- \\
2008\end{array}$ & $\begin{array}{c}37.1 \% \\
(587 / 1,581)\end{array}$ & CNPq \\
\hline $\begin{array}{l}\text { Traebert } \\
\text { et al. } 50 \\
(2010)\end{array}$ & Palhoça (SC) & $\begin{array}{l}\text { Two-phase } \\
\text { random } \\
\text { sampling } \neq \neq\end{array}$ & $\begin{array}{l}12 \text { years-old } \\
\text { children living in } \\
\text { the whole city }\end{array}$ & $\begin{array}{c}405 \\
(194+211 \precsim)\end{array}$ & $\begin{array}{l}\text { Public and } \\
\text { private } \\
\text { schools }\end{array}$ & 7 & O'Brien & 2003 & $\begin{array}{c}23.5 \% \\
(95 / 405)\end{array}$ & + \\
\hline $\begin{array}{l}\text { Wendt } \\
\text { et al. } 51 \\
(2010)\end{array}$ & Pelotas (RS) & $\begin{array}{l}\text { Two-phase } \\
\text { random } \\
\text { sampling 执 }\end{array}$ & $\begin{array}{l}\text { 1-5 years-old } \\
\text { preschool } \\
\text { children living in } \\
\text { the whole city }\end{array}$ & $\begin{array}{c}571 \\
\left(278+293{ }^{\Uparrow}\right)\end{array}$ & $\begin{array}{l}\text { Public and } \\
\text { private } \\
\text { schools }\end{array}$ & 3 & Andreasen & 2009 & $\begin{array}{c}36.6 \% \\
(209 / 571)\end{array}$ & CNPq \\
\hline $\begin{array}{l}\text { Goettems } \\
\text { et al. } 52 \\
(2012)\end{array}$ & Pelotas (RS) & $\begin{array}{l}\text { Two-phase } \\
\text { random } \\
\text { sampling 执 }\end{array}$ & $\begin{array}{l}\text { 2-5 years-old } \\
\text { preschool } \\
\text { children living in } \\
\text { the whole city }\end{array}$ & $\begin{array}{c}501 \\
(2429259 \gtrsim)\end{array}$ & $\begin{array}{l}\text { Public and } \\
\text { private } \\
\text { schools }\end{array}$ & 3 & Andreasen & 2007 & $\begin{array}{c}39.5 \% \\
(198 / 501)\end{array}$ & + \\
\hline
\end{tabular}

(continues) 
Table 1 (continued)

\begin{tabular}{|c|c|c|c|c|c|c|c|c|c|c|}
\hline $\begin{array}{l}\text { Region/ } \\
\text { Study (year } \\
\text { of publi- } \\
\text { cation) }\end{array}$ & City (State) & $\begin{array}{l}\text { Sampling } \\
\text { method }\end{array}$ & $\begin{array}{l}\text { Represen- } \\
\text { tativity }\end{array}$ & $\begin{array}{c}\text { Sample } \\
\text { analyzed (n) }\end{array}$ & Setting & $\begin{array}{l}\text { Number } \\
\text { of raters }\end{array}$ & $\begin{array}{l}\text { Dental } \\
\text { trauma } \\
\text { diagnostic } \\
\text { classifi- } \\
\text { cation } \\
\text { criteria }\end{array}$ & $\begin{array}{c}\text { Year of } \\
\text { data } \\
\text { colle- } \\
\text { ction }\end{array}$ & $\begin{array}{c}\text { Prevalence } \\
\text { of dental } \\
\text { trauma }\end{array}$ & $\begin{array}{l}\text { Funding } \\
\text { source }\end{array}$ \\
\hline $\begin{array}{l}\text { Damé- } \\
\text { Teixeira } \\
\text { et al. } 53 \\
\text { (2013) }\end{array}$ & $\begin{array}{c}\text { Porto Alegre } \\
\text { (RS) }\end{array}$ & $\begin{array}{l}\text { Two-phase } \\
\text { random } \\
\text { sampling \& }\end{array}$ & $\begin{array}{l}12 \text { years-old } \\
\text { children living in } \\
\text { the whole city }\end{array}$ & $\begin{array}{c}1,528 \\
(758 \text { + } 770 \precsim)\end{array}$ & $\begin{array}{l}\text { Public and } \\
\text { private } \\
\text { schools }\end{array}$ & 1 & O'Brien & 2010 & $\begin{array}{c}34.8 \% \\
(531 / 1,528)\end{array}$ & CAPES \\
\hline $\begin{array}{l}\text { Goettems } \\
\text { et al. } 54 \\
(2014)\end{array}$ & Pelotas (RS) & $\begin{array}{l}\text { Two-phase } \\
\text { random } \\
\text { sam- } \\
\text { pling 执 }\end{array}$ & $\begin{array}{l}\text { 8-12 years-old } \\
\text { children living in } \\
\text { the whole city }\end{array}$ & $\begin{array}{c}1,210 \\
\left(6369574{ }^{\Uparrow}\right)\end{array}$ & $\begin{array}{l}\text { Public and } \\
\text { private } \\
\text { schools }\end{array}$ & 6 & O'Brien & 2010 & $\begin{array}{c}12.6 \% \\
(153 / 1,210)\end{array}$ & CNPq \\
\hline $\begin{array}{l}\text { Guedes } \\
\text { et al. } 55 \\
\text { (2014) }\end{array}$ & $\begin{array}{c}\text { Santa Maria } \\
\text { (RS) }\end{array}$ & $\begin{array}{l}\text { Two-phase } \\
\text { random } \\
\text { sampling \&\& }\end{array}$ & $\begin{array}{l}1-5 \text { preschool } \\
\text { children living in } \\
\text { the whole city }\end{array}$ & $\begin{array}{c}478 \\
(2469232 \precsim)\end{array}$ & $\begin{array}{c}\text { National } \\
\text { Child } \\
\text { Vaccination } \\
\text { Day }\end{array}$ & 15 & O'Brien & + & $\begin{array}{c}14.1 \% \\
(66 / 478)\end{array}$ & FAPESP \\
\hline $\begin{array}{l}\text { Agostini } \\
\text { et al. } 56 \\
(2016)\end{array}$ & $\begin{array}{c}\text { Santa Maria } \\
\text { (RS) }\end{array}$ & $\begin{array}{l}\text { Two-phase } \\
\text { random } \\
\text { sampling \&\& }\end{array}$ & $\begin{array}{l}1-5 \text { preschool } \\
\text { children living in } \\
\text { the whole city }\end{array}$ & $\begin{array}{c}\text { 2008: } 455 \\
(2109245 \hat{)}) \\
2010: 639 \\
(318+321 ð) \\
2013: 556 \\
(263+283 ð)\end{array}$ & $\begin{array}{c}\text { National } \\
\text { Child } \\
\text { Vaccination } \\
\text { Day }\end{array}$ & $\begin{array}{l}15 \text { (per } \\
\text { year) }\end{array}$ & O'Brien & $\begin{array}{c}2008, \\
2010 \\
\text { and } \\
2013\end{array}$ & $\begin{array}{c}\text { 2008: } 31.5 \% \\
(139 / 441) \\
2010: 13.0 \% \\
(83 / 639) \\
2013: 22.5 \% \\
(123 / 546)\end{array}$ & $\begin{array}{l}\text { CAPES and } \\
\text { CNPq }\end{array}$ \\
\hline $\begin{array}{l}\text { Comim } \\
\text { et al. } 57 \\
(2021)\end{array}$ & $\begin{array}{c}\text { Santa Maria } \\
\text { (RS) }\end{array}$ & $\begin{array}{l}\text { Single- } \\
\text { phase } \\
\text { random } \\
\text { sampling * }\end{array}$ & $\begin{array}{l}\text { 15-19 years-old } \\
\text { children living in } \\
\text { the whole city }\end{array}$ & $\begin{array}{c}1,197 \\
\left(684+513{ }^{\lambda}\right)\end{array}$ & $\begin{array}{l}\text { Public and } \\
\text { private } \\
\text { schools }\end{array}$ & 2 & O'Brien & 2018 & $\begin{array}{c}17.0 \% \\
(203 / 1,197)\end{array}$ & CAPES \\
\hline \multicolumn{11}{|l|}{ All regions } \\
\hline $\begin{array}{l}\text { Bomfim } \\
\text { et al. } 58 \\
\text { (2017) }\end{array}$ & $\begin{array}{c}\text { All Brazilian } \\
\text { states }\end{array}$ & $\begin{array}{l}\text { Stratified } \\
\text { multi-phase } \\
\text { cluster sam- } \\
\text { pling \&\&\& }\end{array}$ & $\begin{array}{c}12 \text { years old } \\
\text { children of } \\
\text { whole country }\end{array}$ & $\begin{array}{c}7,240 \\
(3,642 q 3,598\end{array}$ & Home & $\begin{array}{c}10 \text { per } \\
\text { capital } \\
\text { and } 2 \\
\text { to } 6 \\
\text { per coun- } \\
\text { tryside } \\
\text { munici- } \\
\text { palities }\end{array}$ & Andreasen & 2010 & $\begin{array}{c}23.9 \% \\
(1735 / 7,240)\end{array}$ & + \\
\hline
\end{tabular}

†: female; ô: male; +: not cited by author; CAPES: Brazilian Graduate Studies Coordinating Board; CNPq: Brazilian National Research Council; FAPEG: Goiás State Research Foundation; FAPEMIG: Minas Gerais State Research Foundation; FAPESP: São Paulo State Research Foundation; GO: Goiás; MG: Minas Gerais; PB: Paraíba; PE: Pernambuco; PR: Paraná; SC: Santa Catarina; SP: São Paulo; RS: Rio Grande do Sul.

* Schoolchildren from all public and private schools were randomly selected using a list provided by the local education authorities;

** Preschools/schools were randomly selected from each health district in the first phase and children were randomly selected from each preschool/ school in the second phase. Sample distribution was proportional to the total population enrolled in private and public preschools in each administrative district of the city;

*** First, the schools were grouped into sampling units of approximately the same size, according to their location, and were chosen by a systematic sampling technique. Then, a second-stage sampling frame including all school classes of the selected sampling units (grouped schools) was created. A total of 114 classes, 19 for each age, was selected using a systematic sampling technique. All the children attending class on the day the researcher visited the school were invited to participate;

\# One municipal health clinics from each region of the city were randomly selected. All children present in the selected health clinic were invited to participate;

\#\# The authors only cited that a stratified cluster sampling method was performed:

\#\# Participants were randomly selected using a systematic sampling procedure, in all 11 health centers of the city. The sample was stratified according to the number of children who had attended each health center in the previous year;

(continues) 


\section{Table 1 (continued)}

$\S$ The sample of schoolchildren was selected in three stages: (1) a sample was randomly selected proportionally to the distribution of the number of schoolchildren in each administrative district of the city; (2) the number of schoolchildren in public and private schools within each administrative district was then used for the estimation of a representative sample; and (3) classes were randomly chosen at each selected school;

$\S \S$ Students in the ninth year of secondary school education and in high school participated in the study. Each class was coded and submitted to randomization using an automated statistics program, thereby ensuring proportionality by grade;

$\S \S \S$ Randomization was performed using systematic sampling. Accordingly, the children were arranged in a line, with the first child examined, the second not examined, the third child examined, and so on;

$\dagger$ All municipal health centers (one of each district of the city) were included. Then, each fifth child in the queue was invited to participate. If parents did not agree to participate, the next child in the queue was selected;

t† The sample was stratified according to the nine administrative districts. The first stage was the randomization of public and private schools in each administrative district of the city. In the second and third stages, classes and children were randomly chosen from the selected schools (proportionally to the total number of children enrolled in the schools);

${ }_{t+t}$ The first-stage units comprised all private and public primary schools in the city. As the number of children differed from school to school, an equal probability scheme was adopted by sampling with probability proportional to size of schools. All children enrolled in the selected schools were invited to participate in the survey;

¥ The health centers were randomly selected, and all children were invited to participate;

¥ First, the schools were grouped into sampling units of approximately the same size and were chosen by a systematic sampling technique. Then, the school children of the selected schools were randomly selected;

$\ddagger \ddagger \ddagger$ The sampling strategy was stratified by type of school: private or public. In each stratum, schools were randomly sampled in a simple way within each stratum. All enrolled children from the selected schools within the age group studied were initially eligible for the study;

\& The sampling strategy was stratified by type of school: private or public. In each stratum, schools were randomly sampled in a simple way within each stratum. Schoolchildren were then randomly selected proportional to the number of schoolchildren in each school;

\&\& 15 health centers that are equally distributed in the five administrative regions of the city were randomly selected. During the survey, every fifth child in the queue for vaccination was invited to participate. If parents did not agree to participate, then the parents of the next child were invited;

\&\&\& The sample of the SB Brasil Project was obtained using a stratified multistage cluster sampling method. Primary sampling units were census tracts for state capitals and the Federal District. Cities were the primary sampling units for interior municipalities. Households within the census tracts for the state capitals and the Federal District and households for the interior municipalities were selected in the second sampling stage.

\section{Synthesis of results, meta-analysis, and regression}

\section{- Prevalence in deciduous teeth}

For this outcome, nine studies 27,31,33,38,40,41,48,52,56 were included in the meta-analysis. Overall, 6,841 children aged between 1 and 6 years were analyzed, and the prevalence of dental trauma in this population was 35\% (95\%CI: 26.0; 44.0; I2 = 98\%). Regarding the results of the subgroup analysis by region, the Northeast presented the higher prevalence of dental trauma (53\%; 95\%CI: 49.0; 56.0) (Figure 2a). Considering the subgroup by sex, the prevalence of dental trauma in boys (40\%; 95\%CI: 29.0; 51.0) was higher than in girls (34\%; 95\%CI: 24.0; 44.0) (Figure 2b), but without significant difference between the subgroups.

Among the variables studied in the meta-regression analysis, we observed that the average age of the sample contributed significantly $(\mathrm{p}<0.0001)$ to explain $82.7 \%$ of the variability. The increase of the average age in 1 year causes an increase of 0.12 in the prevalence of trauma in the deciduous teeth (Figure 3). The other variables studied in the meta-regression did not contribute significantly to the variability between the prevalence: HDI ( $\mathrm{p}=0.6421)$; sampling method $(\mathrm{p}=0.3360)$, and population size $(\mathrm{p}=0.2416)$

Visual inspection of the funnel plot did not reveal asymmetry in the distribution of studies. The Egger test confirmed this finding $(\mathrm{p}=0.8676)$. 


\section{Figure 2}

Subgroup analyses of the prevalence of dental trauma in deciduous teeth.

2a) Brazilian regions

Study

Northeast

Neves et al. ${ }^{27}$

Random effects model

Heterogeneity: not applicable

Southeast

Dutra et al. ${ }^{31}$

Viegas et al. ${ }^{38}$

Corrêa-Faria et al. ${ }^{40}$

Bonini et al. ${ }^{33}$

Tello et al. ${ }^{41}$

Random effects model

Heterogeneity: $\left.\right|^{2} 99 \%, t^{2}=0.0270, p<0.01$

\section{South}

Kramer et al. ${ }^{48}$

Goettems et al. ${ }^{52}$

Agostini et al. ${ }^{56}$

Random effects model

Heterogeneity: $1^{2} 95 \%, t^{2}=0.0187, p<0.01$

\section{Random effects model}

Heterogeneity: $I^{2} 98 \%, \mathrm{t}^{2}=0.0221, \mathrm{p}<0.01$

Test for subgroup differences: $X 23=23.51, d f=2(p<0.01)$

2b) Sex (boys)

Study

Boys

Neves et al. ${ }^{27}$

Dutra et al. ${ }^{31}$

Corrêa-Faria et al. ${ }^{40}$

Bonini et al. ${ }^{33}$

Kramer et al. ${ }^{48}$

Goettems et al. ${ }^{52}$

Random effects model

Heterogeneity: $1^{2} 95 \%, t^{2}=0.0187, p<0.01$

\section{Girls}

Neves et al. ${ }^{27}$

Dutra et al. ${ }^{31}$

Corrêa-Faria et al. ${ }^{40}$

Bonini et al. ${ }^{33}$

Kramer et al. 48

Goettems et al. 52

Random effects model

Heterogeneity: $\mathrm{I}^{2}$ 95\%, $\mathrm{t}^{2}=0.0166, \mathrm{p}<0.01$

\section{Random effects model}

Heterogeneity: $I^{2}$ 95\%, $\mathrm{t}^{2}=0.0164, \mathrm{p}<0.01$

Test for subgroup differences: $\mathrm{X} 21=0.61, \mathrm{df}=1(\mathrm{p}<0.44)$
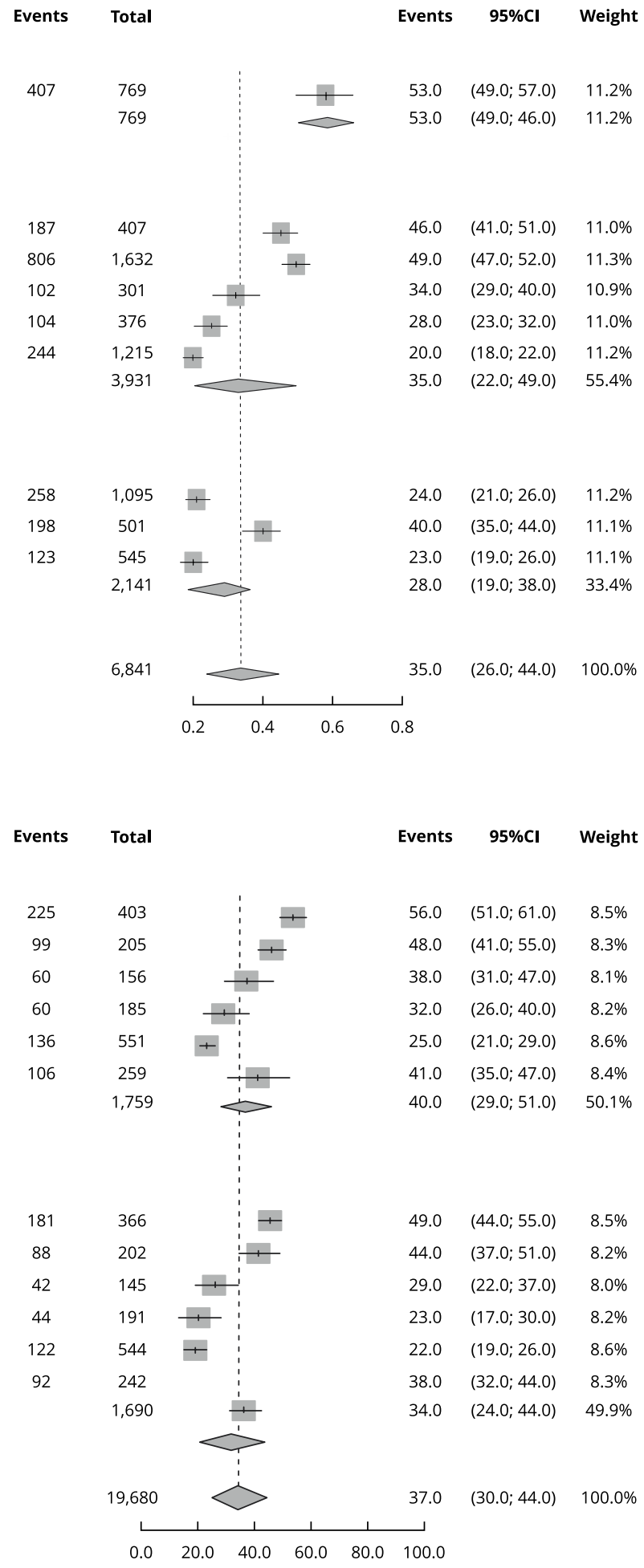


\section{Figure 3}

Meta-regression analysis of the influence of the sample average age in the prevalence of dental trauma in deciduous teeth.

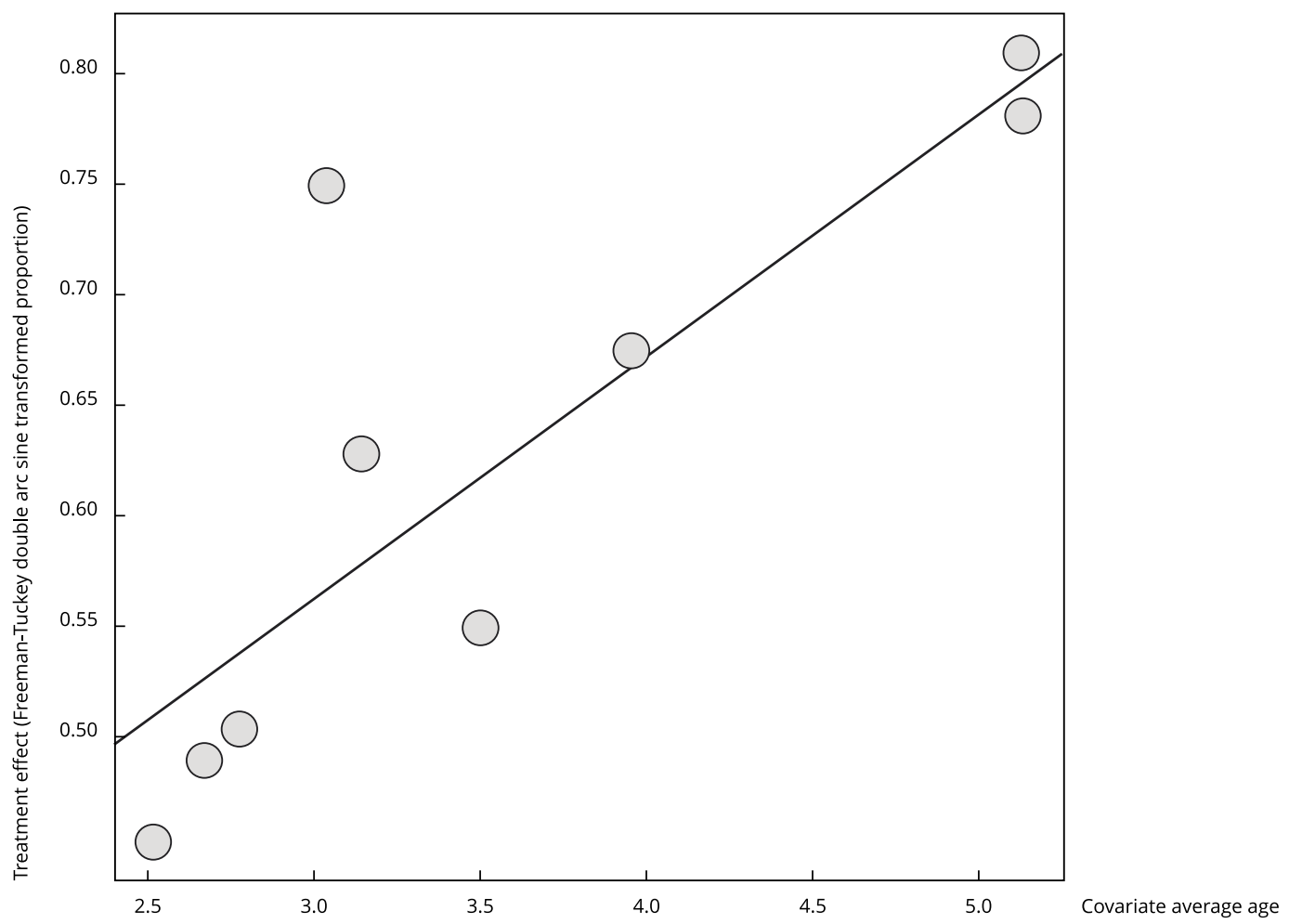

\section{- Prevalence in permanent teeth}

Nineteen studies $14,24,28,29,32,36,37,39,42,43,44,45,46,47,49,50,53,54,57$ were included in this analysis, with a total sample of 19,781 children and adolescents aged between 7 and 19 year old. The prevalence of dental trauma was $21 \%\left(95 \% \mathrm{CI}: 16.0 ; 26.0, \mathrm{I}^{2}=99 \%\right)$. Considering the Brazilian regions, the study shows that the Northeastern (14\%, 95\%CI: 8.0; 22.0, I2 $=96 \%)$ and the Central-West $(17 \%, 95 \%$ CI: 16.0; 19.0, $\mathrm{I}^{2}=$ not available) regions presented the smallest prevalence; however, without statistical difference from Southeastern and Southern regions (Figure 4a). Regarding the variables studied in the meta-regressions, none of them contributed to the variability of the estimates: year of publication $(\mathrm{p}=0.471)$; HDI $(\mathrm{p}=0.528)$; diagnostic criteria $(\mathrm{p}=0.718)$; average age $(\mathrm{p}=0.222)$; and population size $(\mathrm{p}=0.897)$.

Regarding the subgroup analysis by sex, the prevalence of dental trauma in boys (25\%, 95\%CI: $\left.20.0 ; 31.0, I_{2}=98 \%\right)$ was significantly higher than in girls (17\%, 95\%CI: 13.0; 22.0, I2 =97\%) (Figure 4b). None of the variables studied in the meta-regressions contributed to the variability between the prevalence of each subgroup: (1) boys: year of publication $(\mathrm{p}=0.306)$; HDI $(\mathrm{p}=0.796)$; average age $(\mathrm{p}=0.278)$; diagnostic criteria $(\mathrm{p}=0.625)$; region $(\mathrm{p}=0.683)$ and population size $(\mathrm{p}=0.791)$; (2) girls: year of publication $(\mathrm{p}=0.847)$; HDI $(\mathrm{p}=0.918)$; average age $(\mathrm{p}=0.150)$; diagnostic criteria $(\mathrm{p}=0.950)$; region $(\mathrm{p}=0.912)$ and population size $(\mathrm{p}=0.845)$.

Visual inspection of the funnel plot did not reveal asymmetry in the distribution of studies. The Egger test confirmed this finding $(\mathrm{p}=0.514)$. 
Figure 4

Subgroup analyses of the prevalence of dental trauma in permanent teeth.

4a) Brazilian regions

Study

Northeast

Soriano et al. ${ }^{42}$

Random effects model

Heterogeneity: $I^{2} 96 \%, t^{2}=0.0053, p<0.01$

Central-West

Freire et al. ${ }^{2}$

Random elects model

Heterogeneity: not applicable

Southeast

Jorge et al. ${ }^{32}$

Bendo et al. ${ }^{36}$

Freire-Maia et al. ${ }^{14}$

Oliveira Filho et al. ${ }^{35}$

Ramos-Jorge et al. ${ }^{39}$

Random effects model

Heterogeneity: $\left.\right|^{2}$ 97\%, $\mathrm{t}^{2}=0.0100, \mathrm{p}<0.01$

South

Marcenes et al. ${ }^{42}$

Marcenes et al. ${ }^{43}$

Nicolau et al. ${ }^{44}$

Traebert et al. ${ }^{45}$

Traebert et al. ${ }^{46}$

Traebert et al. ${ }^{47}$

Carvalho et al. ${ }^{49}$

Traebert et al. ${ }^{50}$

Damé-Teixeira et al. ${ }^{53}$

Goettems et al. ${ }^{54}$

Comim et al. ${ }^{57}$

Random effects model

Heterogeneity: $I^{2} 97 \%, t^{2}=0.0283, p<0.01$

Random effects mode

Heterogeneity: $I^{2} 99 \%, t^{2}=0.0283, p<0.01$

Test for subgroup differences: $X 23=24.10, d f=3(p<0.25)$
Carvalho et al. ${ }^{28}$

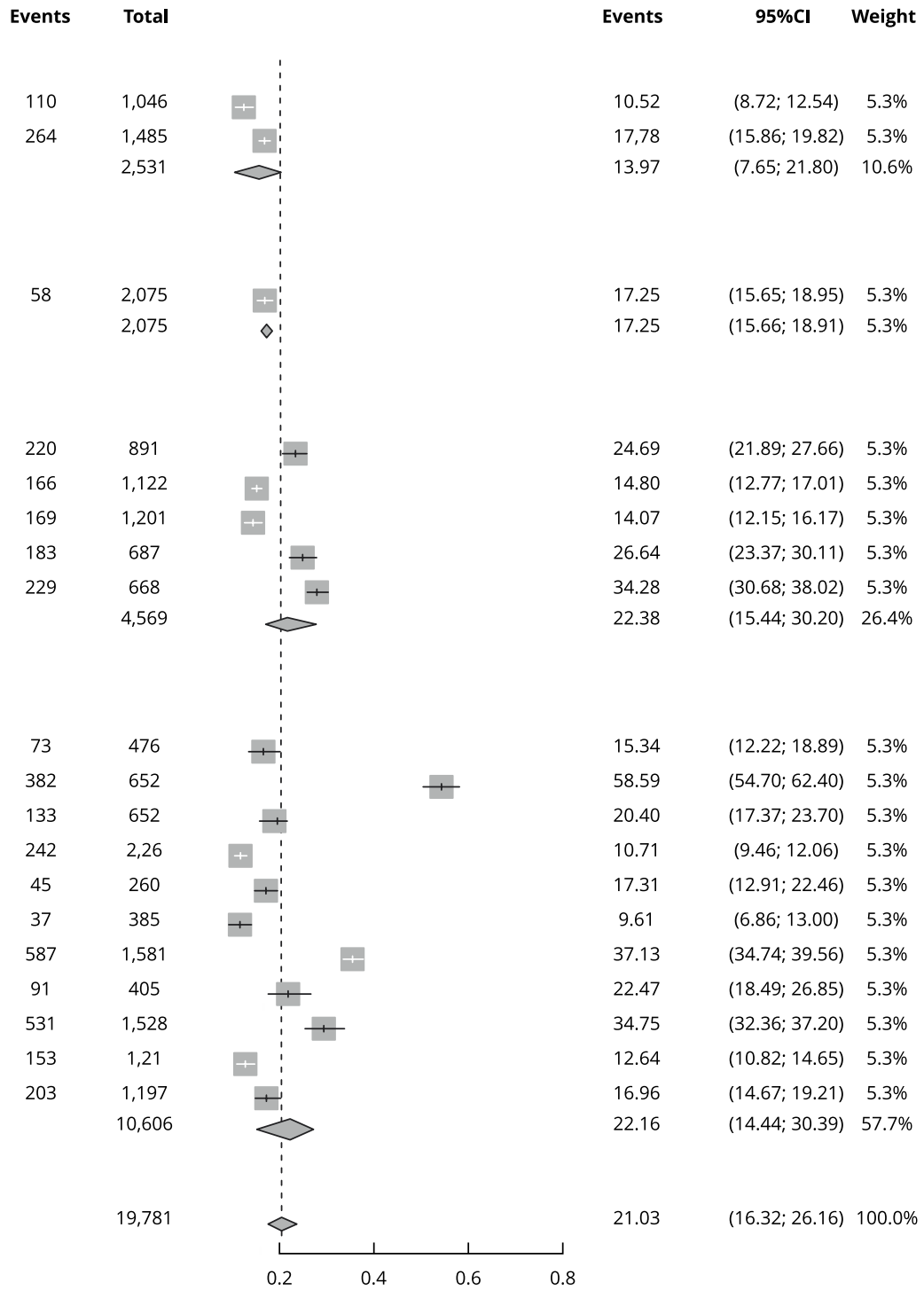

(continues)

\section{- Certainty of evidence}

The certainty of the evidence was classified as "low" for both permanent and deciduous analysis. The certainty of the prevalence of dental trauma in permanent dentition was downgraded in two levels due to inconsistency, while the certainty of the prevalence of dental trauma in deciduous teeth was downgraded in one level due to inconsistency and one level due to imprecision (Supplementary Material. Table S2. http://cadernos.ensp.fiocruz.br/static//arquivo/suppl-e00015920_9970.pdf). 
Figure 4 (continued)

4b) Sex (Girls)

Study

Boys

Soriano et al. ${ }^{42}$

Carvalho et al. ${ }^{28}$

Freire et al. ${ }^{29}$

Jorge et al. ${ }^{32}$

Bendo et al. ${ }^{36}$

Freire-Maia et al. ${ }^{14}$

Oliveira Filho et al. 35

Ramos-Jorge et al. ${ }^{39}$

Marcenes et al. 42

Marcenes et al. ${ }^{43}$

Nicolau et al. ${ }^{44}$

Traebert et al. ${ }^{45}$

Traebert et al. ${ }^{46}$

Traebert et al. ${ }^{47}$

Carvalho et al. ${ }^{49}$

Traebert et al. ${ }^{50}$

Damé-Teixeira et al. 53

Goettems et al. ${ }^{54}$

Comim et al.

Random effects model

Heterogeneity: $\left.\right|^{2}=98 \%, t^{2}=0.0209, p<0.01$

Girls

Soriano et al. ${ }^{42}$

Carvalho et al. ${ }^{28}$

Freire et al. ${ }^{29}$

Jorge et al. ${ }^{32}$

Bendo et al. ${ }^{36}$

Freire-Maia et al. ${ }^{14}$

Oliveira Filho et al. 35

Ramos-Jorge et al. ${ }^{39}$

Marcenes et al. 42

Marcenes et al. ${ }^{43}$

Nicolau et al. 44

Traebert et al. ${ }^{45}$

Traebert et al. ${ }^{46}$

Traebert et al. ${ }^{47}$

Carvalho et al. ${ }^{49}$

Traebert et al. 50

Damé-Teixeira et al. ${ }^{53}$

Goettems et al. 54

Comim et al. ${ }^{57}$

Random effects mode

Heterogeneity: $\left.\right|^{2}=97 \%, t^{2}=0.0 .0155, p<0.01$

Random effects model

Heterogeneity: $\mathrm{l}^{2}=98 \%, \mathrm{t}^{2}=0.0193, \mathrm{p}<0.01$

Test for subgroup differences: $X 21=4.76, d f=1(p=0.03)$

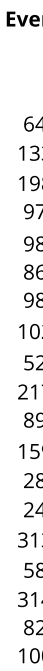

Total

Events

$95 \%$ CI Weight

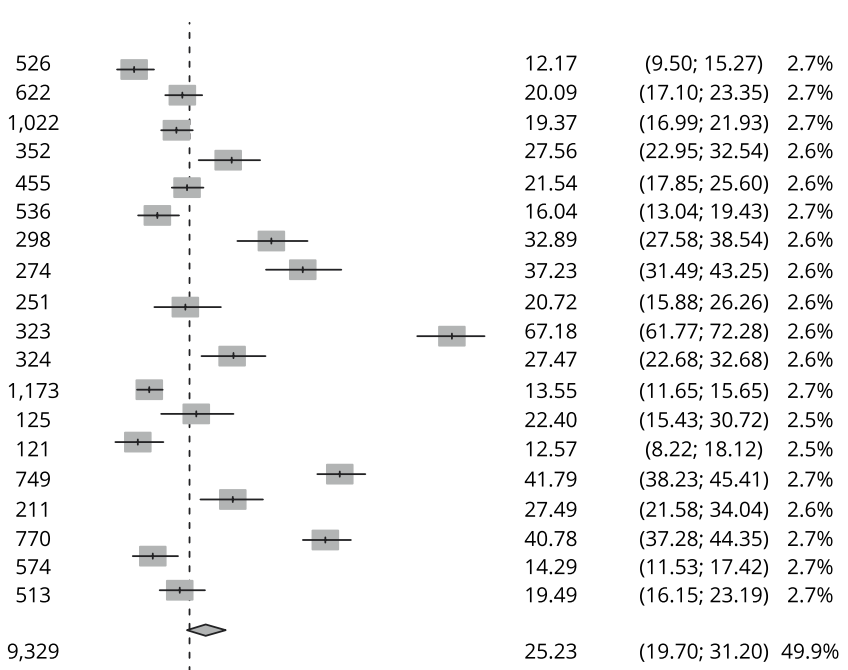

46

131
153

153
123

68

83

85

127

21

165

44

83
17

13

274

33
217

217

104

$(8.13 ; 14.34) \quad 2.6 \%$

$(13.48 ; 18.60) \quad 2.7 \%$

$(12.46 ; 16.80) \quad 2.7 \%$

$(19.34 ; 26.60) \quad 2.7 \%$

$(8.00 ; 12.75) \quad 2.7 \%$

$(10.07 ; 15.24) \quad 2.7 \%$

$(17.89 ; 26.36) \quad 2.6 \%$

$(27.64 ; 37.10) \quad 2.6 \%$

(5.87: 13.91) $2.6 \%$

(44.62; 55.68) $2.6 \%$

$(9.92 ; 17.59) \quad 2.6 \%$

$(6.13 ; 9.38) \quad 2.7 \%$

$(7.51 ; 19.39) \quad 2.5 \%$

(3.62; 11.19) $2.6 \%$

(29.74; 36.24) $2.7 \%$

(12.01; 23.05) $2.6 \%$

(25.43; 31.99) $2.7 \%$

$(8.82 ; 13.87) \quad 2.7 \%$

(12.60; 18.12) $2.7 \%$

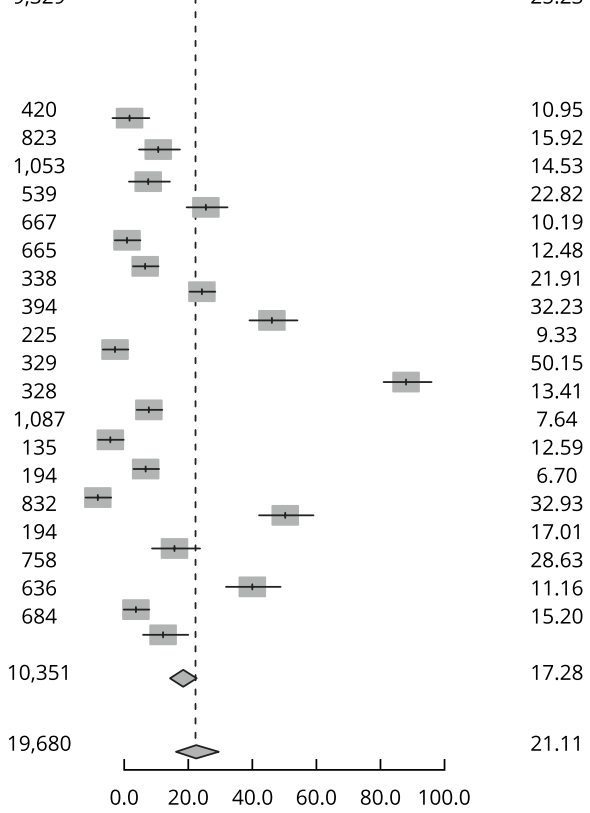

(13.17; 21.82$) 50.1 \%$

\section{(17.55; 24.90) $100.0 \%$}

\section{Discussion}

Being aware of the epidemiological profile of a given disease is the first step in establishing coping policies. dental trauma is a relevant injury that directly affects people's quality of life. This study indicates a considerable dental trauma prevalence among the young population in Brazil, which should be considered a public health problem.

Brazil is a large country, divided into five geographical regions with distinct social, cultural, economic, and natural characteristics. The Brazilian population is concentrated in metropolitan regions near the coast, more specifically in the Southeast, Northeast, and South, where $88 \%$ of the population 
is located 59. Understanding this aspect helps to explain the fact that most studies included in this systematic review were conducted in these particular regions. Plus, despite being the country that publishes the most articles on the prevalence of dental trauma in the world 3 , such publications come from the most populous states with the largest research centers.

However, the knowledge of this unequal distribution of publications is extremely alarming, since almost half of the Brazilian states have not had an assessment of the dental trauma prevalence index. The lack of epidemiological data on dental trauma, especially in the North Region, can lead to unawareness of treatment needs for these conditions caused by possible sequelae, such as tooth loss, pulp necrosis, and decreased quality of life 5 .

One aspect noticed in the eligible studies was the use of different criteria for dental trauma evaluation. In this review, we chose to include only studies that used the criteria of Andreasen or that of O'Brien, as they are the methods used in epidemiological surveys of government agencies. The method of classifying dental trauma proposed by Andreasen is currently the one recommended by the IADT since it is easy to reproduce in addition to having great objectivity in diagnosing traumatic dental injuries. The main difference of the Andreasen criteria from O'Brien's criteria is that tooth discoloration is not used to diagnose dental trauma given that this characteristic may be associated with other pathological changes. The literature about the influence of these criteria on epidemiological results is scarce, and they do not even discuss the possibility of aggregating data from studies that used different methods in meta-analyses. Based on this, we investigated this variable as a possible source of heterogeneity in the estimates of this review; however, the results of the meta-regression showed no association. The current evidence supports the fact that there is no suitable system for establishing the diagnosis of the studied injuries that could be applied to epidemiological surveys since these systems do not evaluate traumatic injuries that can only be diagnosed radiographically 60 .

Furthermore, a significant methodological characteristic of eligible studies that must be highlighted and discussed is the sampling method. In epidemiological studies, random probabilistic sampling from a defined subset of the population (sample frame) should be used in most cases to guarantee the representativeness of the population. In this review, we decided to include only studies that used this sampling method to provide the best evidence possible, which can explain the high number of studies with a low risk of bias included in our qualitative analysis.

The prevalence of dental trauma in permanent teeth was $21 \%$, whereas in deciduous teeth was $35 \%$. Such results are higher than those obtained by Petti et al. 3 , which performed a systematic review with studies from different countries and found a prevalence of $15 \%$ in permanent teeth and $22 \%$ in deciduous teeth. This difference may be explained by methodological differences between these two reviews, and because Petti et al.'s 3 revision included several other countries with socioeconomic, behavioral, and phenotypic differences from Brazil. However, it is important to highlight that in both reviews, the prevalence of dental trauma in deciduous dentition was higher than in permanent dentition, which can be explained by the great vulnerability of younger children to falls and accidents 3 .

When evaluating the subgroup analysis by Brazilian region, we can observe similar prevalence in permanent dentition in the Northeast, Central-West, Southeast, and the South. Similar results were found in the epidemiological survey conducted by the Brazilian Ministry of Health in 2010 55, where it was observed that the prevalence of pediatric dental trauma between regions varied from $18 \%$ to $25 \%$. However, unlike the survey results, this meta-analysis included children and adolescents aged 7 to 19 years. Regarding the prevalence of dental trauma in deciduous dentition, the Northeastern Region presented a significantly higher prevalence than the Southeastern and the Southern region. Yet, the results for prevalence in deciduous teeth in the Northeastern region ought to be interpreted with caution since they correspond to the results of a single study.

The prevalence of dental trauma in boys was higher than in girls in most eligible studies from this review. Also, according to the meta-analysis, the prevalence of trauma among boys follows the same high prevalence. The results agrees with the literature and can be explained by the fact that boys culturally present more hyperactive habits and behaviors in daily life than girls, such as physical contact sports, and consequently, they are more involved in dangerous situations 61,62 .

All meta-analyses in this review presented high heterogeneity. Even though we run a metaregression with several variables, only the heterogeneity in the analysis of the prevalence in deciduous teeth could be partially explained by the mean age of the sample included in the studies. The meta- 
regression shows that the prevalence of dental trauma increases with age; this finding is due to the cumulative characteristic of dental trauma in this age group and was confirmed in a previous systematic review 62. Maybe this variable was not capable of explaining the heterogeneity in the permanent dentition because this age group is exposed to others risk factors that are not directly related to age, such as malocclusions 63 , alcohol or illicit drugs use 64 , or sport practice 65 .

This study has some limitations. The first one is the high and unexplained heterogeneity in some of the analysis. Then, the discrepancy between the number of eligible studies published when comparing the regions of Brazil is another limitation. Furthermore, this study does not explain the causes of dental trauma occurrence, it only indicates the existing prevalence.

On the other hand, the current study is original and contributes to the literature with the following points: it is the first systematic review with meta-analysis of the literature to evaluate the prevalence of dental trauma in different regions of Brazil, considering the type of dentition and sex of the population. Plus, it is also noteworthy that publication bias was not observed in any analysis. So, this finding can be attributed to the extensive research performed in databases, without restrictions on language, year, and publication status. Moreover, this review encompassed a much larger number of studies than previews systematic reviews of prevalence.

Overall, it is important to keep in mind that dental trauma is a public health problem, and it must be prevented. Subsequently, well-designed studies are needed to indicate which preventive actions must be developed to reduce the dental trauma occurrence and their effect on the quality of life of children and adolescents.

\section{Conclusion}

The prevalence of dental trauma among Brazilian children and adolescents is higher than that found worldwide, both in the permanent and primary dentition, and is similar throughout the Brazilian regions. Also, the prevalence of dental trauma among boys is higher than in girls. 


\section{Contributors}

W. A. Vieira conceived the idea, contributed substantially to the study design, data analysis and interpretation, writing of the manuscript, final approval of the manuscript and is also guarantor of the study, responsible for the integrity of the work as a whole, from inception to the published article. V. G. A. Pecorari participated on meta-analysis, data analysis, drafting and revision of the manuscript, and its final approval. R. Figueiredo-de-Almeida, J. F. A. Almeida and A. de-Jesus-Soares contributed substantially to the study design, data analysis and interpretation, writing of the manuscript, and its final approval. J. Vargas-Neto, E. C. A. Santos and B. P. F. A. Gomes contributed substantially to data analysis and interpretation, and the writing of the manuscript and final approval of the manuscript. N. Carvas Junior participated on data analysis and interpretation, writing and review of the manuscript, and its final approval.

\section{Additional informations}

ORCID: Walbert de Andrade Vieira (0000-00018872-2865); Vanessa Gallego Arias Pecorari (00000002-0300-5697); Rodolfo Figueiredo-de-Almeida (0000-0003-2072-5458); Nelson Carvas Junior (0000-0003-2168-8927); Júlio Vargas-Neto (00000001-8170-615X); Eduardo César Almada Santos (0000-0003-4116-2535); Brenda Paula Figueiredo de Almeida Gomes (0000-0002-8449-0646); José Flávio Affonso de Almeida (0000-0002-5874-9247); Adriana de-Jesus-Soares (0000-0002-8078-1606).

\section{References}

1. Levin L, Day PF, Hicks L, O'Connell A, Fouad $\mathrm{AF}$, Bourguignon $\mathrm{C}$, et al. International Association of Dental Traumatology guidelines for the management of traumatic dental injuries: general introduction. Dent Traumatol 2020; 36:309-13.

2. Petti S, Andreasen JO, Glendor U, Andersson L. The fifth most prevalent disease is being neglected by public health organizations. Lancet Glob Health 2018; 6:e1070-1.

3. Petti S, Glendor U, Andersson L. World traumatic dental injury prevalence and incidence, a meta-analysis: one billion living people have had traumatic dental injuries. Dent Traumatol 2018; 34:71-86.

4. Aldrigui JM, Jabbar NS, Bonecker M, Braga MM, Wanderley MT. Trends and associated factors in prevalence of dental trauma in Latin America and Caribbean: a systematic review and meta-analysis. Community Dent Oral Epidemiol 2014; 42:30-42.

5. Zaror C, Martínez-Zapata MJ, Abarca J, Díaz J, Pardo Y, Pont A, et al. Impact of traumatic dental injuries on quality of life in preschoolers and schoolchildren: a systematic review and meta-analysis. Community Dent Oral Epidemiol 2018; 46:88-101.

6. Carvalho V, Jacomo DR, Campos V. Frequency of intrusive luxation in deciduous teeth and its effects. Dent Traumatol 2010; 26:304-7.

7. Souza BDM, Dutra KL, Kuntze MM, Bortoluzzi EA, Flores-Mir C, Reyes-Carmona J, et al. Incidence of root resorption after the replantation of avulsed teeth: a meta-analysis. J Endod 2018; 44:1216-27.

8. Rocha Lima TF, Nagata JY, Souza-Filho FJ, Soares AJ. Post-traumatic complications of severe luxations and replanted teeth. J Contemp Dent Pract 2015; 16:13-9.

9. Lee JY, Divaris K. Hidden consequences of dental trauma: the social and psychological effects. Pediatr Dent 2009; 31:96-101.

10. Secretaria de Atenção a Saúde, Secretária de Vigilância em Saúde, Ministério da Saúde. SB Brasil 2010. Pesquisa Nacional de Saúde Bucal: resultados principais. Brasília: Ministério da Saúde; 2012.

11. Felix CP, Shitsuka C, Moriyama CM, Duarte DA. Exploratory study of the prevalence of traumatic injuries in preschool children in the city of Macapá, Brazil. Pesqui Bras Odontopediatria Clín Integr 2014; 14:71-7.

12. Lira ALS, Dias LPS, Martins CWC, Santos TCS. Prevalence and etiology of dental trauma in schoolchildren aged 6 to 12 years. Braz J Oral Sci 2018; 17:e18082.

13. Frujeri ML, Frujeri JA, Bezerra AC, Cortes MI, Costa Jr. ED. Socio-economic indicators and predisposing factors associated with traumatic dental injuries in schoolchildren at Brasília, Brazil: a cross-sectional, population-based study. BMC Oral Health 2014; 14:91. 
14. Freire-Maia FB, Auad SM, Abreu MHNG, Sardenberg F, Martins MT, Paiva SM, et al. Prevalence of and factors associated with enamel fracture and other traumas in Brazilian children 8-10 years old. Braz Oral Res 2018; 32:e89.

15. Todero SRB, Cavalcante-Leão BL, Fraiz FC, Rebellato NLB, Ferreira FM. The association of childhood sleep problems with the prevalence of traumatic dental injury in schoolchildren. Dent Traumatol 2019; 35:41-7.

16. Page MJ, McKenzie JE, Bossuyt PM, Boutron I, Hoffmann TC, Mulrow CD, et al. The PRISMA 2020 statement: an updated guideline for reporting systematic reviews. BMJ 2021; 372:n71.

17. Aromataris E, Munn Z. Joanna Briggs Institute reviewer's manual. Adelaide: Joanna Briggs Institute; 2017.

18. World Health Organization. Oral health surveys: basic methods. 5th Ed. Geneva: World Health Organization; 2013.

19. Silva MF, Leite FRM, Ferreira LB, Pola NM, Scannapieco FA, Demarco FF, et al.. Estimated prevalence of halitosis: a systematic review and meta-regression analysis. Clin Oral Investig 2018; 22:47-55.

20. Polmann H, Melo G, Conti Réus J, Domingos FL, Souza BDM, Padilha AC, et al. Prevalence of dentofacial injuries among combat sports practitioners: a systematic review and metaanalysis. Dent Traumatol 2020; 36:124-40.

21. Klotz T, Munn Z, Aromataris EC, Greenwood JE. Imiquimod to prevent keloid recurrence postexcision: a systematic review and metaanalysis. Wound Repair Regen 2020; 28:145-56.

22. Balshem $H$, Helfand $M$, Schünemann HJ, Oxman AD, Kunz R, Brozek J et al. GRADE guidelines. 3. Rating the quality of evidence. J Clin Epidemiol 2013; 64:401-6.

23. Iorio A, Spencer FA, Falavigna M, Alba C, Lang E, Burnand B, et al. Use of GRADE for assessment of evidence about prognosis: rating confidence in estimates of event rates in broad categories of patients. BMJ 2015; 350:h870.

24. Soriano EP, Caldas Jr. AF, Carvalho MV, Caldas KU. Relationship between traumatic dental injuries and obesity in Brazilian schoolchildren. Dent Traumatol 2009; 25:506-9.

25. Siqueira MB, Gomes MC, Oliveira AC, Martins CC, Granville-Garcia AF, Paiva SM. Predisposing factors for traumatic dental injury in primary teeth and seeking of post-trauma care. Braz Dent J 2013; 24:647-54.

26. Gomes MC, Pinto-Sarmento TC, Costa EM, Martins CC, Granville-Garcia AF, Paiva SM. Impact of oral health conditions on the quality of life of preschool children and their families: a cross-sectional study. Health Qual Life Outcomes 2014; 12:55.

27. Neves ETB, Perazzo MF, Gomes MC, Martins CC, Paiva SM, Granville-Garcia AF. Perception of parents and self-reports of children regarding the impact of traumatic dental injury on quality of life. Dental Traumatol 2017; 33:444-50.
28. Carvalho B, Almeida H, Andrade ESS, Zarzar P, Vieira SCM, Heimer MV, et al. Prevalence of dental trauma in 1,485 Brazilian adolescents aged between 15 and 19 years old and associated factors. Oral Health Prev Dent 2020; 18:707-12.

29. Freire MC, Vasconcelos DN, Vieira AS, Araujo JA, Moreira RS, Nunes MF. Association of traumatic dental injuries with individual-, sociodemographic- and school-related factors among schoolchildren in Central-West Brazil. Int J Environ Res Public Health 2014; 11:9885-96.

30. Cortes MI, Marcenes W, Sheiham A. Prevalence and correlates of traumatic injuries to the permanent teeth of schoolchildren aged 9-14 years in Belo Horizonte, Brazil. Dent Traumatol 2001; 17:22-6.

31. Dutra FT, Marinho AM, Godoi PF, Borges CM, Ferreira EF, Zarzar PM. Prevalence of dental trauma and associated factors among 1- to 4-year-old children. J Dent Child (Chic) 2010; 77:146-51.

32. Jorge KO, Oliveira Filho PM, Ferreira EF, Oliveira AC, Vale MP, Zarzar PM. Prevalence and association of dental injuries with socioeconomic conditions and alcohol/drug use in adolescents between 15 and 19 years of age. Dent Traumatol 2012; 28:136-41.

33. Bonini GC, Bönecker M, Braga MM, Mendes FM. Combined effect of anterior malocclusion and inadequate lip coverage on dental trauma in primary teeth. Dent Traumatol 2012; 28:437-40

34. Ramos-Jorge ML, Ramos-Jorge J, Mota-Veloso I, Oliva KJ, Zarzar PM, Marques LS. Parents' recognition of dental trauma in their children. Dent Traumatol 2013; 29:266-71.

35. Castro FC, Raggio DP, Imparato JCP, Piovesan C, Bonini GC. Impact of oral problems on the quality of life of preschool children. Pesqui Bras Odontopediatria Clín Integr 2013; 13:361-9.

36. Bendo CB, Paiva SM, Abreu MH, Figueiredo LD, Vale MP. Impact of traumatic dental injuries among adolescents on family's quality of life: a population-based study. Int J Paediatr Dent 2014; 24:387-96.

37. Oliveira Filho PM, Jorge KO, Paiva PCP, Ferreira EFE, Ramos-Jorge ML, Zarzar PM. The prevalence of dental trauma and its association with illicit drug use among adolescents. Dent Traumatol 2014; 30:122-7.

38. Viegas CM, Paiva SM, Carvalho AC, Scarpelli AC, Ferreira FM, Pordeus IA. Influence of traumatic dental injury on quality of life of Brazilian preschool children and their families. Dent Traumatol 2014; 30:338-47.

39. Ramos-Jorge J, Paiva SM, Tataounoff J, Pordeus IA, Marques LS, Ramos-Jorge ML. Impact of treated/untreated traumatic dental injuries on quality of life among Brazilian schoolchildren. Dent Traumatol 2014; 30:27-31.

40. Corrêa-Faria P, Paiva SM, Pordeus IA, RamosJorge ML. Influence of clinical and socioeconomic indicators on dental trauma in preschool children. Braz Oral Res 2015; 29:1-7. 
41. Tello G, Bonini GC, Murakami C, Abanto J, Oliveira LB, Bonecker M. Trends in the prevalence of traumatic crown injuries and associated factors in Brazilian preschool children: 10-year observational data. Dent Traumatol 2016; 32:274-80.

42. Marcenes W, Alessi ON, Traebert J. Causes and prevalence of traumatic injuries to the permanent incisors of school children aged 12 years in Jaragua do Sul, Brazil. Int Dent J 2000; 50:87-92.

43. Marcenes W, Zabot NE, Traebert J. Socioeconomic correlates of traumatic injuries to the permanent incisors in schoolchildren aged 12 years in Blumenau, Brazil. Dent Traumatol 2001; 17:222-6.

44. Nicolau B, Marcenes W, Sheiham A. The relationship between traumatic dental injuries and adolescents' development along the life course. Community Dent Oral Epidemiol 2003; 31:306-13.

45. Traebert J, Almeida IC, Garghetti C, Marcenes W. Prevalence, treatment needs, and predisposing factors for traumatic injuries to permanent dentition in 11-13-year-old schoolchildren. Cad Saúde Pública 2004; 20:403-10.

46. Traebert J, Bittencourt DD, Peres KG, Peres MA, Lacerda JT, Marcenes W. Aetiology and rates of treatment of traumatic dental injuries among 12-year-old school children in a town in southern Brazil. Dent Traumatol 2006; 22:173-8

47. Traebert J, Hemkemeier I, Lacerda JT. Traumatismo em dentes permanentes recém-irrompidos: prevalência e fatores associados em escolares do município de Tubarão - SC. Rev Odont UNESP 2008; 37:363-9.

48. Kramer PF, Gomes CS, Ferreira SH, Feldens CA, Viana EdS. Traumatismo na dentição decídua e fatores associados em pré- escolares do município de Canela/RS. Pesqui Bras Odontopediatria Clín Integr 2009; 9:95-100.

49. Carvalho ML, Moyses SJ, Bueno RE, Shimakura S, Moyses ST. A geographical population analysis of dental trauma in school-children aged 12 and 15 in the city of Curitiba-Brazil. BMC Health Serv Res 2010; 10:203.

50. Traebert J, Marcon KB, Lacerda JT. Prevalence of traumatic dental injuries and associated factors in schoolchildren of Palhoça, Santa Catarina State. Ciênc Saúde Colet 2010; 15 Suppl 1:1849-55.

51. Wendt FP, Torriani DD, Assunção MC, Romano AR, Bonow ML, Costa CT, et al. Traumatic dental injuries in primary dentition: epidemiological study among preschool children in South Brazil. Dent Traumatol 2010; 26:168-73.

52. Goettems ML, Azevedo MS, Correa MB, Costa CT, Wendt FP, Schuch HS, et al. Dental trauma occurrence and occlusal characteristics in Brazilian preschool children. Pediatr Dent 2012; 34:104-7.

53. Damé-Teixeira N, Alves LS, Susin C, Maltz M. Traumatic dental injury among 12-year-old South Brazilian schoolchildren: prevalence, severity, and risk indicators. Dent Traumatol 2013; 29:52-8.
54. Goettems ML, Torriani DD, Hallal PC, Correa MB, Demarco FF. Dental trauma: prevalence and risk factors in schoolchildren. Community Dent Oral Epidemiol 2014; 42:581-90.

55. Guedes RS, Piovesan C, Antunes JL, Mendes FM, Ardenghi TM. Assessing individual and neighborhood social factors in child oral health-related quality of life: a multilevel analysis. Qual Life Res 2014; 23:2521-30.

56. Agostini BA, Pinto LT, Koehler M, Emmanuelli B, Piovesan C, Ardenghi TM. Trend of traumatic crown injuries and associated factors in preschool children. Braz Oral Res 2016; 30:e112.

57. Comim LD, Dalla Nora A, Knorst JK, Racki DNO, Zenkner JEDA, Alves LS. Traumatic dental injury and oral health-related quality of life among 15 to 19 year old adolescents from Santa Maria, Brazil. Dent Traumatol 2021; 37:58-64.

58. Bomfim RA, Herrera DR, De-Carli AD. Oral health-related quality of life and risk factors associated with traumatic dental injuries in Brazilian children: a multilevel approach. Dent Traumatol 2017; 33:358-68.

59. Instituto Brasileiro de Geografia e Estatística. Sinopse do Censo Demográfico 2010. Rio de Janeiro: Instituto Brasileiro de Geografia e Estatística; 2011.

60. Feliciano KM, Caldas Jr. AF. A systematic review of the diagnostic classifications of traumatic dental injuries. Dent Traumatol 2006; 22:71-6.

61. Soares TRC, Magno MB, Jural LA, Loureiro JM, Chianca TK, Risso PA, et al. Risk factors for traumatic dental injuries in the Brazilian population: a critical review. Dent Traumatol 2018; 34:445-54.

62. Corrêa-Faria P, Martins CC, Bönecker M, Paiva SM, Ramos-Jorge ML, Pordeus IA. Clinical factors and socio-demographic characteristics associated with dental trauma in children: a systematic review and meta-analysis. Dent Traumatol 2016; 32:367-78.

63. Arraj GP, Rossi-Fedele G, Doğramacı EJ. The association of overjet size and traumatic dental injuries: a systematic review and meta-analysis. Dent Traumatol 2019; 35:217-32.

64. Magno MB, Leite KLF, Pithon MM, Maia LC. Are traumatic dental injuries greater in alcohol or illicit drugs consumers? A systematic review and meta-analysis. Drug Alcohol Depend 2019; 197:236-49.

65. Werlich MO, Honnef LR, Bett JVS, Domingos FL, Pauletto P, Souza BDM, et al. Prevalence of dentofacial injuries in contact sports players: a systematic review and meta-analysis. Dent Traumatol 2020; 36:477-88. 


\section{Resumo}

O estudo buscou investigar a prevalência de trauma dentário em crianças e adolescentes brasileiros. Foi realizada uma revisão sistemática em oito bases de dados: MEDLINE (via PubMed), LILACS, BBO, Scopus, Embase, Web of Science, Open Access Theses and Dissertations e OpenThesis. Foram incluidos apenas estudos de prevalência que usavam métodos de amostragem probabilistica, sem limitação de ano ou idioma de publicação. Para avaliar o risco individual de viés, foram utilizadas as ferramentas de avaliação crítica da JBI para estudos de prevalência. Os estudos individuais foram combinados na metanálise com o uso do modelo de efeitos aleatórios. A heterogeneidade entre os estudos foi analisada pelas estatísticas $Q$ de Cochran e I-quadrado. A análise de metarregressão foi realizada para avaliar as fontes de heterogeneidade. A abordagem GRADE avaliou a certeza das evidências entre os estudos incluídos. A busca resultou em 2.069 registros, dos quais 36 foram incluídos no estudo. Os estudos elegíveis foram publicados entre 2000 e 2021, com uma amostra total de 40.194 crianças e adolescentes. A maioria dos estudos (75\%) teve baixo risco de viés. Nos dentes permanentes, a prevalência de trauma dentário foi de 21\% (IC95\%: 16,0; 26,0) e nos dentes decíduos foi de 35\% (IC95\%: 26,0; 44,0). A prevalência de trauma dentário foi mais alta no sexo masculino que no feminino, para ambas as dentições. Com base na baixa certeza, a prevalência das lesões dentárias traumáticas em crianças $e$ adolescentes brasileiros é mais alta que no resto do mundo, tanto nos dentes decíduos quanto nos permanentes. Além disso, a prevalência de trauma dentário é mais alta em meninos que em meninas.

Traumatismos Dentários; Criança; Adolescente; Revisão Sistemática

\section{Resumen}

Este estudio tuvo como meta investigar la prevalencia de trauma dental en niños y adolescentes brasileños. Se realizó una revisión sistemática considerando ocho bases de datos: MEDLINE (via PubMed), LILACS, BBO, Scopus, Embase, Web of Science, Open Access Theses and Dissertations $y$ OpenThesis. Solamente se incluyeron estudios de prevalencia que usaron un método de muestreo probabilístico, sin restricción sobre el año o lengua de publicación. Se usó las herramientas de evaluación crítica del JBI para estudios de prevalencia studies para evaluar el riesgo individual de sesgo. Los estudios individuales se combinaron en metaanálisis, usando un modelo de efectos aleatorios. La heterogeneidad entre los estudios se analizó mediante las estadisticas de Cochran Q e I-cuadrado. Se realizó un análisis de meta-regresión para evaluar las fuentes de heterogeneidad. El enfoque GRADE evaluó la certidumbre de evidencia a través de los estudios incluidos. La búsqueda resultó en 2.069 registros, de los cuales treinta y seis se incluyeron en el estudio. Los estudios elegibles se publicaron entre 2000 y 2021, con una muestra total de 40.194 niños y adolescentes. La mayoría de los estudios (75\%) tenían un bajo riesgo de sesgo. En los dientes permanentes la prevalencia de trauma dental fue 21\% (IC95\%: 16,0; 26,0) y en los dientes deciduos la prevalencia de trauma dental fue $35 \%$ (IC95\%: 26,0; 44,0). La prevalencia trauma dental entre niños fue más alta que entre niñas en ambas denticiones. Basado en una baja certidumbre, la prevalencia de lesiones traumáticas dentales en niños y adolescentes brasileños es más alta que la encontrada en el resto del mundo, tanto en dientes deciduos como en dientes permanentes. Asimismo, la prevalencia de trauma dental entre niños es más alta que entre niñas.

Traumatismos de los Dientes; Niño; Adolescente; Revisión Sistemática
Submitted on $31 / \mathrm{Jan} / 2020$

Final version resubmitted on 26/May/2021

Approved on 06/Jul/2021 